Pure and Applied Mathematics Quarterly

Volume 4, Number 2

(Special Issue: In honor of

Fedor Bogomolov, Part 1 of 2)

$319-361,2008$

\title{
From Solitons to Many-Body Systems
}

David Ben-Zvi and Thomas Nevins

\begin{abstract}
We present a bridge between the KP soliton equations and the Calogero-Moser many-body systems through noncommutative algebraic geometry. The Calogero-Moser systems have a natural geometric interpretation as flows on spaces of spectral curves on a ruled surface. We explain how the meromorphic solutions of the KP hierarchy have an interpretation via a noncommutative ruled surface. Namely, we identify KP Lax operators with vector bundles on quantized cotangent spaces (formulated technically in terms of $\mathcal{D}$-modules). A geometric duality (a variant of the Fourier-Mukai transform) then identifies the parameter space for such vector bundles with that for the spectral curves and sends the KP flows to the Calogero-Moser flows. It follows that the motion and collisions of the poles of the rational, trigonometric and elliptic solutions of the KP hierarchy, as well as of its multicomponent analogs, are governed by the (spin) Calogero-Moser systems on cuspidal, nodal and smooth genus one curves. This provides geometric explanations and generalizations of results of Airault-McKean-Moser, Krichever and Wilson. The present paper is an overview of work to appear in [BN2].
\end{abstract}

\section{INTRODUCTION}

Our purpose in this paper is to introduce a geometric viewpoint (developed in detail in [BN2]) on a much-explored, puzzling phenomenon of the theory of integrable systems: the description of the motion of poles of meromorphic solutions of soliton equations by simple many-body systems. Our viewpoint provides

Received February 27, 2006. 
a conceptual framework for this phenomenon within noncommutative algebraic geometry. As an application we obtain direct geometric proofs of results that complete the work of Airault-McKean-Moser [AMM], Krichever [Kr1, Kr2] and Wilson [W3] on the motions and collisions of poles of meromorphic KP solutions.

1.1. Historical Overview. Before reviewing the Calogero-Moser system (CM), the Kadomtsev-Petviashvili hierarchy (KP), and their relation, we sketch an incomplete historical overview of the problem - see the review articles [Be, GW] for more complete history and bibliography. In the seminal work [AMM], Airault, McKean and Moser wrote down rational, trigonometric and elliptic solutions of the Korteweg-deVries equation and discovered that the motion of their poles is governed by the Calogero-Moser classical many-body systems of particles on the line, cylinder and torus (respectively) with inverse square potentials. Krichever $[\mathrm{Kr} 1, \mathrm{Kr} 2]$ and the Chudnovskys [CC] extended this correspondence to the meromorphic solutions of the KP equation, where it becomes an isomorphism between the phase spaces of generic rational (decaying at infinity), trigonometric and elliptic KP solitons and the corresponding Calogero-Moser systems.

Krichever derived this result (in the elliptic case) from a relation between an auxiliary linear problem associated to a KP potential and the CalogeroMoser systems. More precisely, to an elliptic KP potential one associates a nonstationary Schrödinger operator with elliptic potential, and Krichever proved that this auxiliary Schrödinger operator has meromorphic solutions if and only if the poles of the potential move as particles in the elliptic CM system. In particular, this showed that the elliptic CM system can be written in terms of spectral curves (using a Lax operator with spectral parameter), and showed that the generic elliptic solutions of KP are finite gap solutions - they come from applying Krichever's general geometric construction of solutions to KP to these spectral curves, known as tangential covers of the elliptic curve. A detailed algebro-geometric study of tangential covers was undertaken by Treibich and Verdier [TV1, TV2], leading to a complete classification of elliptic solutions of the KdV equation. This geometric description of the elliptic CM systems may be used to identify them with a meromorphic version of the Hitchin system [GN, Ne1, DW, DM] — for the rational and trigonometric systems, the corresponding Hitchin systems live on cuspidal or nodal (rather than smooth) genus one curves [Ne1] (see also [Kr5] for a different 
point of view on this identification, closer in spirit to the current work, as part of the theory of meromorphic Lax operators on curves).

Other work on the $\mathrm{KP} / \mathrm{CM}$ correspondence includes generalizations to some cases of multicomponent KP hierarchy and spin Calogero-Moser systems [BBKT, $\mathrm{T} 1$ ], an analog for difference equations relating the 2D Toda hierarchy and Ruijsenaars-Schneider systems [KrZ, T2, Kr3, Kr4], and extensions to other related systems (see e.g. [Kr3, Kr4, GW, BB, BrH]). The $\mathrm{KP} / \mathrm{CM}$ correspondence is also applied in the study of the bispectral phenomenon [DG, W1, W2, W3, W4, BW1, W5] and relates to Seiberg-Witten integrability of supersymmetric Yang-Mills theory (see $[\mathrm{BrK}]$ for a collection of reviews).

1.2. Some Recent Developments. The $\mathrm{KP} / \mathrm{CM}$ correspondence in the rational case was greatly deepened by Wilson [W3] (see [W5] for a review), who extended it to allow collisions of particles. More precisely, the rational CalogeroMoser phase space possesses a natural completion that is constructed as a space of pairs of matrices whose commutator lies in a particular conjugacy class. In this description, the positions of the particles correspond to the eigenvalues of one of the matrices, which are now allowed to coincide. Wilson had (see [W1]) identified the completed phase space of the rational KP hierarchy with an adelic Grassmannian that parametrizes certain subspaces of $\mathbf{C}[x]$. In [W3], Wilson gives an explicit formula that defines a point of the adelic Grassmannian from a CM pair of matrices and then proves by direct calculations that this map extends continuously to the completed phase spaces and takes the CM flows to the KP flows. (In the generic rational case, Shiota [Shi] has previously extended the $\mathrm{KP} / \mathrm{CM}$ correspondence to all the higher flows of the KP hierarchy and the higher $\mathrm{CM}$ hamiltonians, establishing a bijection between generic rational solutions of the KP hierarchy and the Calogero-Moser hierarchy; see also the related work $[\mathrm{AKV}])$.

Wilson's adelic Grassmannian appears independently in the work of Cannings and Holland [CH1] classifying (right) ideals in the Weyl algebra of differential operators on the affine line, indicating that there might be an interesting relationship between the $\mathrm{KP} / \mathrm{CM}$ correspondence and objects of noncommutative algebra/geometry. In [BW1] (see [W4] for a review), Berest and Wilson show that the decomposition of the rational KP phase space into the union of $n$-particle CM spaces can be described as the orbit decomposition under the action of the group 
of automorphisms of the Weyl algebra, "half" of which is matched up with the KP flows. This decription is then used to identify Wilson's bispectral involution [W1] (see [W2] for a review) on the rational KP solutions with the Fourier transform on the Weyl algebra.

Inspired by ideas of Le Bruyn [LB], Berest and Wilson [BW2] explored a direct relation, which was extended by Baranovsky, Ginzburg, and Kuznetsov [BGK1], between the Calogero-Moser pairs of matrices and the classification of right ideals in the Weyl algebra through a calculation in noncommutative geometry. Namely, by interpreting the ideals as sheaves on a noncommutative projective plane, one can apply the techniques of Koszul duality and the Beilinson spectral sequence to classify ideals (up to isomorphism as modules) by cohomological data (monads), which turns out to reproduce precisely the Calogero-Moser matrices.

However, a direct relation between KP and ideals in the Weyl algebra (or noncommutative geometry) was missing, as were a conceptual explanation of the relation between KP and Calogero-Moser systems and an extension of the correspondence to completed phase spaces in the trigonometric, elliptic and multicomponent cases. These goals are achieved in [BN2], and described in the present paper.

1.3. The Current Work. As we have already indicated, the present work is devoted to to introducing a geometric approach to the $\mathrm{KP} / \mathrm{CM}$ correspondence that is developed in detail in [BN2]. Accordingly, we begin with a review (Section 2) of the (rational, trigonometric and elliptic) Calogero-Moser systems and their concrete construction (by pairs of matrices, in the rational case). We then describe the formulation [TV2] of the Calogero-Moser systems as flows on the space of pairs $(\Sigma, \mathcal{L})$ of a line bundle $\mathcal{L}$ on a curve $\Sigma$ (the spectral curve), embedded in a ruled surface $E^{\natural}$ over a (cuspidal, nodal or smooth) genus 1 curve $E$. To pass between this geometric picture and the concrete Calogero-Moser particles, we recall the identification of both with a special case of the meromorphic Hitchin system on $E$.

In Section 3 we discuss the KP hierarchy, its Lax formulation in terms of formal microdifferential operators, and Sato's reformulation of KP Lax operators in terms of $\mathcal{D}$-modules. Sato identifies Lax operators with a quotient of an open subset (the big cell $\mathrm{Gr}^{\circ}$ ) of an infinite-dimensional Grassmannian Gr. We introduce a coordinate-free reformulation of meromorphic KP Lax operators, the 
micro-opers, by generalizing the $\mathcal{D}$-modules appearing in Sato's theory. We then extend (Theorem 4.7) Sato's identification between a quotient of the big cell of the Grassmannian and the set of Lax operators on the disc to an identification of a quotient of the entire Grassmannian with the space of micro-opers on the disc. Micro-opers on a general curve $X$ define meromorphic Lax operators on $X$, whose poles correspond to the points where the "local data" lie outside the big cell of the Sato Grassmannian. Thus micro-opers are well-suited to describing the poles of Lax operators and their collisions. The formulation of the KP hierarchy in terms of (regular) micro-opers is closely analogous to the Drinfeld-Sokolov formulation of the KdV hierarchies in terms of connections - indeed, micro-opers are the KP analogues of the opers of Beilinson-Drinfeld ([BD1]) (or more precisely of the affine opers of $[\mathrm{BF}])$.

It is at this stage that noncommutative algebraic geometry enters the picture: we explain in Section 4 how a micro-oper on a curve $X$ may be interpreted as a line bundle or rank one torsion free sheaf on a noncommutative ruled surface, (the completion of) the quantized cotangent bundle $T_{\hbar}^{*} X$ of $X$, equipped with some data along a divisor "at infinity". In this setting micro-differential operators arise geometrically as Laurent expansions of functions on the noncommutative surface along this divisor. Moreover, there is a simple geometric description of the KP flows directly on the space of micro-opers: in terms of the noncommutative ruled surface, the flows act as modifications of bundles along the divisor by "changing the transition function."

In Section 5 we explain how a geometric Fourier transform provides the KP/CM correspondence. Laumon [La2] and Rothstein [Ro2] enhanced the original FourierMukai transform for abelian varieties to a geometric integral transform that takes $\mathcal{D}$-modules on an abelian variety $A$ to quasicoherent sheaves on a bundle $\widehat{A}^{\natural}$ over the dual abelian variety $\widehat{A}$. This enhanced Fourier-Mukai transform was then used in work of Nakayashiki [N1, N2] and Rothstein [Ro1, Ro2] to describe the algebro-geometric solutions of KP. We extend the enhanced Fourier-Mukai transform to the case of singular genus one curves (see Theorem 5.2) and use it to construct an isomorphism between moduli spaces of vector bundles on noncommutative ruled surfaces over genus one curves and moduli spaces of spectral sheaves on a (commutative) ruled surface. This gives a new proof of the classification [BW2, BGK1] of ideals in the Weyl algebra by quiver data, the Calogero-Moser pairs of matrices (this follows from the rank one, rational case of our result). The 
same technique in a degenerate case gives a new proof of the ADHM classification of framed torsion-free sheaves on $\mathbb{P}^{2}$ by quiver data.

Our main result, described in Section 5.3, states that the Fourier transform precisely identifies the phase space of KP solutions on a genus one curve with the spectral data of the CM system, and the KP flows on the former with the CM system on the latter:

Theorem 1.1 ([BN2]). The completed phase spaces of the rational, trigonometric, and elliptic Calogero-Moser systems are identified, via the extended FourierMukai transform, with the moduli spaces of rational, trigonometric, and elliptic $K P$ Lax operators (taken up to change of coordinate in $\partial^{-1}$ ). This isomorphism identifies the KP and CM hierarchies. The poles of the micro-opers correspond to the positions of Calogero-Moser particles, extending the bijection of [Kr1, Kr2] in the generic case and that of [W3] in the completed rational case.

The KP solutions with generic singularities, which are identified with configurations of $\mathrm{CM}$ particles with distinct positions in [Kr1, Kr2], correspond to micro-opers which only hit the codimension one strata of the Sato Grassmannian. The collisions of CM particles correspond to passing to more interesting singularities of the micro-opers, i.e. deeper strata of the Grassmannian. In particular, we obtain that the correspondence extends to completed phase spaces for the rational, trigonometric and elliptic systems, generalizing Wilson's result [W3] in the rational one-component case. (Here Wilson's adèlic Grassmannian appears naturally in its Cannings-Holland interpretation, as a parameter space of $\mathcal{D}$-modules.)

The same technique, applied to higher rank vector bundles or micro-opers, identifies the completed phase spaces of the multicomponent generalizations of $\mathrm{KP}$ with those for the spin generalizations of the Calogero-Moser systems, as is described in [BN2].

We conclude in Section 6 with brief comments on further work on the relation with $\mathcal{W}_{1+\infty}$ vertex algebras [BN6], the bispectral involution of Wilson, and generalization of the results of [BN2] to the difference analog (Toda/Ruijsenaars correspondence) [BN5].

1.4. Calogero-Moser Particles as Points on a Noncommutative Surface.

We have already discussed the role of noncommutative geometry in a geometric 
formulation of meromorphic solutions of KP. However, Theorem 1.1 also has an interesting noncommutative geometric interpretation that is close in spirit to familiar commutative constructions.

Namely, recall that the Hilbert scheme of points on the cotangent bundle $T^{*} X$ of a curve $X$ is isomorphic to (a component of) the moduli space of rank one torsion-free sheaves on the smooth projective completion $\overline{T^{*} X}=T^{*} X \cup X$ that are framed (trivialized) along the divisor at infinity. When one deforms $T^{*} X$ to the quantized cotangent bundle $T_{\hbar}^{*} X$ of $X$ it is this latter description of the Hilbert scheme that deforms well; consequently one takes, as the Hilbert scheme of points on $T_{\hbar}^{*} X$, the moduli space of framed rank one torsion-free sheaves on the projective completion $\overline{T_{\hbar}^{*} X}$ (see [BN4] for more on the relationship to the Hilbert scheme of $\left.T^{*} X\right)$. Note that every micro-oper on $X$ gives such a framed rank one torsion-free sheaf on $\overline{T_{\hbar}^{*} X}$. On the other hand, the natural phase space for a manybody system (such as the CM system) on an elliptic curve $E$ is the configuration space for collections of points on the cotangent bundle $T^{*} E$ with distinct $E$ coordinates. We may seek to complete the phase space (and Hamiltonians) so as to allow collisions of particles. An obvious candidate for a completed phase space would be the Hilbert scheme of points on the cotangent bundle. The natural dynamical system on the Hilbert scheme of points on $T^{*} E$, however, is the trivial many-body system, with zero potential between distinct particles.

In this language our result says that the Calogero-Moser system extends to the Hilbert scheme of points on the quantized cotangent bundle of the curve. While the quantized cotangent bundle does not have any points in the ordinary sense (since $\mathcal{D}_{E}$ has no finite-dimensional modules), the space of micro-opers provides a natural candidate for this Hilbert scheme. We thus show that the natural dynamics of "points on the quantized cotangent bundle" of $E$ (that is, of microopers on $E$ ) is the KP system, and identify this precisely with the Calogero-Moser system. Moreover the positions of the Calogero-Moser particles are easily read off from the micro-oper (as the $E$-coordinates of the putative points in $T_{\hbar}^{*} E$ ), and are identified with the poles of KP Lax operators.

From this point of view, both KP and Calogero-Moser systems naturally live on the quantized cotangent bundle - the interpretation using spectral curves via the Fourier-Mukai transform is then a tool to describe the relevant moduli spaces and check that we are indeed getting the correct Hamiltonian system. 
1.5. Acknowledgements. We would like to thank Ron Donagi, Dennis Gaitsgory, Victor Ginzburg and Tony Pantev for very helpful conversations. We are especially grateful to Edward Frenkel and Mitch Rothstein for generously sharing their understanding of the geometry of soliton equations. Both authors were supported during the course of this work by NSF postdoctoral fellowships, and by MSRI postdoctoral fellowships as part of the Spring 2002 programs "Algebraic Stacks, Intersection Theory and Non-Abelian Hodge Theory" and "InfiniteDimensional Algebras and Mathematical Physics."

\section{Calogero-Moser Systems}

We begin by reviewing the complexified Calogero-Moser systems associated with the general linear group $G L_{n}$, following [KKS, W3, Ne1]. See also [BN3] for a treatment of the CM system adapted to our present needs.

2.1. The CM System. Recall that connected one-dimensional complex algebraic groups $\mathbb{G}$ fall into three classes: the additive group $\mathbb{G}_{a}=\mathbf{C}$, the multiplicative group $\mathbb{G}_{m}=\mathbf{C}^{\times}$, and the one-parameter family of elliptic curves $E$. We denote the identity element of each of these groups by $o$. The complexified Calogero-Moser systems are completely integrable Hamiltonian systems describing a collection of $n$ identical particles on one of these groups $\mathbb{G}$. Thus the phase space of the Calogero-Moser system on the curve $\mathbb{G}$ is $T^{*}\left(\mathbb{G}^{(n)} \backslash \bigcup\{\right.$ all diagonals $\left.\}\right)$, the configurations of $n$ distinct unlabelled points $q_{i} \in \mathbb{G}$ with momenta $p_{i} \in \mathbf{C}$. They are described, in terms of coordinates on the complex line (the universal cover of $\mathbb{G}$ ) by the Hamiltonians

$$
H=\frac{1}{2} \sum_{i=1}^{n} p_{i}^{2}+\sum_{i<j} U\left(q_{i}-q_{j}\right)
$$

with potential functions with a single second order pole at the origin of $\mathbb{G}$, that is

Rational $: U(q)=\frac{1}{q^{2}} \quad$ Trigonometric $: U(q)=\frac{1}{\sin ^{2}(q)} \quad$ Elliptic $: U(q)=\wp(q)$ where $\wp(q)$ is the Weierstrass $\wp$-function attached to the elliptic curve $E$. 
2.1.1. The rational and trigonometric Calogero-Moser systems can be described concretely by Hamiltonian reduction for the group $G L_{n}$ ([KKS]). Let $\mathbb{O} \subset \mathfrak{g l}_{n}$ denote the conjugacy class of traceless matrices of the form $\mathrm{Id}+A$ with $A$ a rank one matrix (which we consider as a coadjoint orbit in $\mathfrak{g l}_{n}^{*}$ ). It is convenient to realize $\mathbb{O}$ as the orbit of either of the matrices

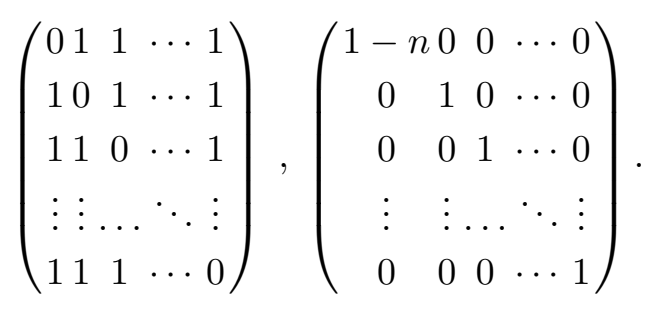

Then the Hamiltonian reduction of $T^{*} \mathfrak{g l}_{n}$ by $G L_{n}$ with the moment condition $\mathbb{O}$ is the space

$$
\mathfrak{C M}_{n}=\left\{(X, Y) \in T^{*} \mathfrak{g l}_{n} \mid[X, Y] \in \mathbb{O}\right\} / G L_{n} .
$$

As proven in [W3], this space is a smooth irreducible affine variety of dimension $2 n$. This variety comes equipped with the Hamiltonian function $H=\frac{1}{2} \operatorname{tr} Y^{2}$, and all other invariant polynomials of $Y$ give Hamiltonians in involution with $H$, whence one deduces the complete integrability of the Calogero-Moser system by a dimension count. On the open subset where $X$ has distinct eigenvalues, we may write coordinates $\left(q_{i}, p_{i}\right)$ on (a finite cover of) $\mathfrak{C M}_{n}$, using the first matrix representative of $\mathbb{O}$ in 2.1 :

$$
X=\left(\begin{array}{ccccc}
q_{1} & 0 & 0 & \cdots & 0 \\
0 & q_{2} & 0 & \cdots & 0 \\
0 & 0 & q_{3} & \cdots & 0 \\
\vdots & \vdots & \cdots & \ddots & \vdots \\
0 & 0 & 0 & \cdots & q_{n}
\end{array}\right), \quad Y=\left(\begin{array}{cccccc}
p_{1} & \frac{1}{q_{1}-q_{2}} & \frac{1}{q_{1}-q_{3}} & \cdots & \frac{1}{q_{1}-q_{n}} \\
\frac{1}{q_{2}-q_{1}} & p_{2} & \frac{1}{q_{2}-q_{3}} & \cdots & \frac{1}{q_{2}-q_{n}} \\
\frac{1}{q_{3}-q_{1}} & \frac{1}{q_{3}-q_{2}} & p_{3} & \cdots & \frac{1}{q_{3}-q_{n}} \\
\vdots & \vdots & \cdots & \ddots & \vdots \\
\frac{1}{q_{n}-q_{1}} & \frac{1}{q_{n}-q_{2}} & \frac{1}{q_{n}-q_{3}} & \cdots & p_{n}
\end{array}\right)
$$

It is easy to see that the Hamiltonian $H$ in these coordinates recovers the rational Calogero-Moser Hamiltonian above. Thus $\mathfrak{C M}_{n}$ provides a completion of the phase space of the rational Calogero-Moser system, in which we allow the points $q_{i}$ (the eigenvalues of $X$ ) to collide. The trigonometric Calogero-Moser system can be described similarly from reduction of $T^{*} G L_{n}$ : we consider conjugacy classes of pairs of matrices $(X, Y)$ with $X$ invertible and $X Y X^{-1}-Y \in \mathbb{O}$. As before, we obtain explicit coordinates on the locus where $X$ is diagonalizable and the commuting Hamiltonians from the invariant polynomials of $Y$. In the next section 
we will see how the Calogero-Moser rank one matrices arise naturally from the consideration of spectral curves over a singular elliptic curve.

2.2. Calogero-Moser Spectral Curves. In this section we present the geometric construction of the Calogero-Moser systems in terms of spectral curves on an algebraic surface (see [TV1, TV2, GN, DW, DM] for the elliptic case, and [Ne1] for the rational and trigonometric cases). The connection with the explicit description of the previous section is explained in Section 2.3 by interpreting both as Hitchin systems.

A general paradigm for constructing integrable systems (see [DM] for a discussion and references) involves fixing a symplectic algebraic surface and a family of curves (specifically, a linear series) on this surface. The phase space of the associated integrable system is the space of pairs $(\Sigma, \mathcal{L})$ of a curve $\Sigma$ in the family and a line bundle (or, for singular $\Sigma$, a rank one torsion-free sheaf) $\mathcal{L}$ on $\Sigma$ (i.e. the family of generalized Jacobians of the curves in the linear series). The Hamiltonians of the system are given by coordinates on the space of curves $\Sigma$, and the flows of the system are linear flows on the Jacobians, modifying $\mathcal{L}$ while fixing $\Sigma$.

In the case of the rational, trigonometric and elliptic Calogero-Moser systems, the relevant symplectic surface $E^{\natural}$ is a special affine bundle over a cuspidal, nodal or smooth elliptic curve $E$, respectively. Recall that an irreducible reduced algebraic curve $E$ of arithmetic genus one, i.e. a Weierstrass cubic curve, falls into one of three classes:

- Elliptic: $E$ is a smooth elliptic curve (in particular a group), and may be described by an equation of the form $y^{2}=x^{3}+a x+b$.

- Trigonometric: $E=\overline{\mathbb{G}}_{m}$ is a nodal cubic, and is isomorphic to the curve $y^{2}=x^{2}(x-1)$. Its normalization $\mathbf{P}^{1} \rightarrow E$ identifies two points 0 and $\infty$ to a node on $E$, and defines a group structure $\mathbb{G}_{m} \cong E^{s m} \subset E$ on the smooth locus.

- Rational: $E=\overline{\mathbb{G}}_{a}$ is a cuspidal cubic, and is isomorphic to the curve $y^{2}=x^{3}$. Its normalization $\mathbf{P}^{1} \rightarrow E$ collapses $2 \cdot \infty$ to a cusp on $E$, and defines a group structure $\mathbb{G}_{a} \cong E^{s m} \subset E$ on the smooth locus.

In each of the cases we have canonical isomorphisms $E^{s m}=\mathbb{G} \cong \mathrm{Pic}^{0} E$ of the corresponding group $\mathbb{G}$ with the Jacobian of $E$, and of $E$ itself with the 
compactified Jacobian of $E$, the moduli of torsion free sheaves of rank one and degree zero on $E$ (trivialized at the identity). ${ }^{1}$

An elliptic curve $E$ carries a unique nontrivial rank 1 affine bundle $E^{\natural} \rightarrow E$. Let At denote the Atiyah bundle on $E$, the unique nontrivial extension of $\mathcal{O}_{E}$ by itself. The (Stein) algebraic surface $E^{\natural}$ may be identified with the complement of the unique section $E_{\infty}=\mathbb{P}(\mathcal{O}) \cong E$ of the projectivization of the Atiyah bundle, $E^{\natural} \subset \bar{E}^{\natural}=\mathbb{P}($ At $) \subset E_{\infty} . E^{\natural}$ is also naturally identified with the moduli space of line bundles with a holomorphic connection on $E .^{2}$ Finally, $E^{\natural}$ may also be identified as a twisted cotangent bundle of $E$, namely as the affine bundle of connections on the line bundle $\mathcal{O}(o)$ on $E$. In particular it inherits a symplectic form.

For a singular cubic curve, one defines an affine bundle $E^{\natural} \rightarrow E$ with analogous properties, taking account of the behavior at the singularity (effectively replacing the tangent sheaf of the curve by its trivial line subbundle generated by the invariant vector field on the group $\mathbb{G}$ ) - see [BN2] for a detailed account. In each case, the projective bundle $\bar{E}^{\natural} \rightarrow E$ has a unique section $E_{\infty}$, whose complement is the affine bundle $E^{\natural}$.

The open (Stein) surface $E^{\natural}$ does not contain any complete curves, so that any curve in $\bar{E}^{\natural}$ must intersect the divisor $E_{\infty}$. So it is natural to consider the simplest linear series on $E^{\natural}$, consisting of curves with only one point of intersection with $E_{\infty}$ (taken for convenience to be the origin $o \in E_{\infty}$ ), and degree $n$ over $E$ for some positive integer $n$. This linear series (denoted $\left|n \cdot E_{\infty}+F\right|$ ) on $\bar{E}^{\natural}$ is studied in detail in [TV1]. In particular, it is equivalent for a curve $\Sigma \in\left|n \cdot E_{\infty}+F\right|$ to be irreducible and to have a unique point of intersection with the section $E$ at infinity (which is then automatically transversal). Moreover the collection of such curves $\Sigma$ is the complement of a hyperplane in the projective space $\left|n \cdot E_{\infty}+F\right|$. There is also a bijection between such $\Sigma \hookrightarrow \bar{E}^{\natural} \rightarrow E$ and tangential covers of $E$, corresponding to maps $E \rightarrow$ Jac $\Sigma$ which are tangent at $o$ to the Abel-Jacobi line of $\Sigma$ at the distinguished point $\Sigma \cap E_{\infty}$.

Thus we consider the integrable system, the completed Calogero-Moser system, with phase space given by line bundles, and more generally rank one torsion-free sheaves, supported on such curves:

\footnotetext{
${ }^{1}$ In particular, there is a universal sheaf $\mathcal{P} \rightarrow E \times E$, the Poincaré sheaf.

${ }^{2}$ The pullback of the Poincaré bundle to $E \times E^{\natural}$ carries a canonical connection relative to the second factor.
} 
Definition 2.1. Let $\mathcal{O}_{o}$ denote the skyscraper sheaf at $o$. A $C M$ spectral sheaf is a coherent sheaf $\mathcal{L}$ on $\bar{E}^{\natural}$ of pure dimension one, equipped with an isomorphism $\phi:\left.\mathcal{L}\right|_{E_{\infty}} \rightarrow \mathcal{O}_{o}$, and with first Chern class $n \cdot E_{\infty}+F$ (where $F$ denotes the fiber over $o) .{ }^{3}$ The space of CM spectral sheaves is denoted by $\mathfrak{C M}_{n}(E)$.

The Hamiltonian flows of the completed Calogero-Moser system preserve the underlying curve $\Sigma$ while modifying the spectral sheaf (linearly) along the generalized Jacobian of $\Sigma$. We may generate all such flows by modifying $\mathcal{L}$ only at the distinguished point $o$ at infinity (as in $[\mathrm{BL}, \mathrm{BF}]$ ). Let $(\mathcal{L}, \Sigma) \in \mathfrak{C M}_{n}(E)$ be a Calogero-Moser spectral sheaf, and let $\mathcal{K}_{\Sigma, \infty}$ denote the field of Laurent series along $\Sigma$ at $\Sigma \cap E_{\infty}$. Then there is a canonical identification $\operatorname{End}\left(\mathcal{L} \otimes \mathcal{K}_{\Sigma, \infty}\right)=$ $\mathcal{K}_{\Sigma, \infty}$. It easily follows that the abelian Lie algebra $\mathcal{K}_{\Sigma, \infty}$ acts (linearly and formally transitively) on the compactified Picard of $\Sigma$ by formally changing the transition function of $\mathcal{L}$ at $\Sigma \cap E_{\infty}$.

2.3. CM and Hitchin. In this section we sketch the description of the CalogeroMoser systems as Hitchin systems following [Ne1, DM] (see also [BN3]).

Let $B u n_{n}(E, o)$ denote the moduli space of rank $n$ semistable vector bundles of degree zero on a cubic curve $E$, equipped with a trivialization of the fiber at the identity; for singular $E$, we impose the (open) condition that the bundle have trivial pullback to the normalization $\mathbf{P}^{1}$. The cotangent fiber $\left.T^{*} B u n_{n}(E, o)\right|_{V}$ at a bundle $V$ consists of pairs $(V, \eta)$, where $\eta$ is a meromorphic Higgs field $\eta \in \Gamma($ End $V(o))$ on $V$ with only a simple pole at $o$. We may perform Hamiltonian reduction of $T^{*} \operatorname{Bun}_{n}(E, o)$ with respect to the action of $\mathrm{GL}_{n}$ changing the trivialization at $o$, with moment map taking values in an arbitrary coadjoint orbit in $\mathfrak{g l}_{n}$. Thus we define the Calogero-Moser-Hitchin space by

$$
T^{*} \operatorname{Bun}_{n}(E, o) / / \mathbb{O} \mathrm{GL}_{n}=\left\{(V, \eta): \operatorname{res}_{o}(\eta) \in \mathbb{O}\right\} / \mathrm{GL}_{n},
$$

that is, as the space of Higgs bundles on $E$ having residue at $o$ in the CalogeroMoser orbit $\mathbb{O}$. The description as a reduction endows the Calogero-MoserHitchin space with a symplectic form and with $n$ algebraically independent Poisson commuting Hamiltonians given by the invariant polynomials in $\eta$ (the Hitchin hamiltonians).

\footnotetext{
${ }^{3}$ One must also impose (generic) normalization conditions, see [BN2, Definition 2.3]. Moreover, if $E$ is singular we add the technical condition that $M$ is locally free over the singular point.
} 
In the rational and trigonometric cases one has concrete descriptions of the moduli spaces: since our bundles have trivial pullback to the normalization $\mathbf{P}^{1}$, they are completely described by the descent data from $\mathbf{P}^{1}$ to $E$. This descent data in the nodal case is the identification of the two fibers over the inverse image of the node, hence $\operatorname{Bun}_{n}\left(\overline{\mathbb{G}}_{m}, o\right)=\mathrm{GL}_{n}$. In the cuspidal case these two points are infinitesimally nearby, and the descent data becomes a "connection matrix" identifying these two nearby fibers - thus we have $B u n_{n}\left(\overline{\mathbb{G}}_{a}, o\right)=\mathfrak{g l}_{n}$. It follows immediately that the Calogero-Moser-Hitchin space for $\overline{\mathbb{G}}_{a}$ is isomorphic with the completed rational Calogero-Moser phase space $\mathfrak{C M}_{n}$ introduced in Section 2.1.1, and similarly in the trigonometric case. In the elliptic case we may recover the elliptic Calogero-Moser particles and Hamiltonians as follows. There is a dense open subvariety of $\operatorname{Bun}_{n}(E, o)$ that is identified with the configuration space of $n$ distinct points $q_{i}$ on $E$, via the assignment

$$
\left\{q_{i}\right\} \mapsto \oplus \mathcal{O}\left(q_{i}-o\right) .
$$

Writing the Hitchin Hamiltonian in these coordinates gives the elliptic CalogeroMoser flow on the $q_{i}$.

Hitchin's integrable system on a curve $X$ is naturally described in terms of spectral curves, this time embedded in the symplectic surface $T^{*} X$. In our case, the minimal (rank one) condition for the matrix $A$ in the coadjoint orbit condition on the Calogero-Moser matrices $[X, Y]=\mathrm{Id}+A$ matches up precisely with the minimal (one-point) intersection condition on the Calogero-Moser spectral curves, yielding the following:

Proposition 2.2. The n particle Calogero-Moser phase space on $E$ is canonically identified with an open subset of the space $\mathfrak{C M}_{n}(E)$ of Calogero-Moser spectral sheaves with support of degree $n$ over $E$. Under this isomorphism, the CM flows are identified with linear flows along the generalized Jacobians of CM spectral curves.

Remark 2.3. See [DW] for a geometric description of this identification via the CM Hitchin system. One begins by describing the meromorphic Hitchin system in terms of spectral curves $\widetilde{\Sigma}$ in the total space of the line bundle $\Omega_{E}(0)=\mathcal{O}_{E}(0)$ of differentials with simple pole at 0 . One then obtains a $\mathrm{CM}$ spectral curve through a birational transformation, blowing up the point in the fiber over 0 corresponding to the eigenvalue 1 , and blowing down the proper transform of the fiber. 


\section{The KP Hierarchy}

In this section we briefly review the definition of the KP hierarchy and its interpretation via flows on the Sato Grassmannian. General references for this section are $[\mathrm{Mu} 2],[\mathrm{S}]$ and $[\mathrm{SW}]$.

3.1. Introducing KP. Let $\mathcal{E}$ denote the algebra of formal microdifferential operators (or pseudodifferential symbols) with coefficients in $\mathbf{C}[[t]]$. An element of $\mathcal{E}$ is a Laurent series

$$
M=\sum_{N \ll \infty} a_{N} \partial^{N} \quad a_{i} \in \mathbf{C}[[t]]
$$

in the formal inverse $\partial^{-1}$ of the derivation $\partial=\partial_{t}$ of $\mathbf{C}[[t]]$. The composition in $\mathcal{E}$ is determined by the Leibniz rule,

$$
\partial^{n} \cdot f=\sum_{i \geq 0}\left(\begin{array}{l}
n \\
i
\end{array}\right) f^{(i)} \partial^{n-i},
$$

where $\left(\begin{array}{c}n \\ i\end{array}\right)$ is defined for $n<0$ by taking

$$
\left(\begin{array}{c}
n \\
i
\end{array}\right)=\frac{n(n-1) \cdots(n-i+1)}{i(i-1) \cdots 2 \cdot 1} .
$$

The ring $\mathcal{D}=\mathbf{C}[[t]]\langle\partial\rangle$ of differential operators is a subring of $\mathcal{E}$. We also have a commutative subring $\Gamma=\mathbf{C}\left(\left(\partial^{-1}\right)\right)$ of constant coefficient microdifferential operators.

Consider a microdifferential operator of the form

$$
L=\partial+u_{1} \partial^{-1}+u_{2} \partial^{-2}+\cdots \in \mathcal{E},
$$

which is called a KP Lax operator. The space $\mathcal{L}$ of such operators is an infinitedimensional affine space (with coordinates the coefficients of the $u_{i}$ ). The KP hierarchy is the collection of compatible evolution equations on a Lax operator $L$ defined as follows:

$$
\frac{\partial L}{\partial t_{n}}=\left[L,\left(L^{n}\right)_{+}\right]
$$

where $(M)_{+}=\sum_{N \geq 0} a_{N} \partial^{N} \in \mathcal{D} \subset \mathcal{E}$ denotes the differential part of a microdifferential operator $M$ as in (3.1). That is, we let the operator $L=L\left(t, t_{1}, t_{2}, \ldots\right)$ depend on the infinitely many time variables $t_{n}$ and then require that the dependence of $L$ on $t_{n}$ (i.e. its "evolution along the $n$th time") satisfies (3.3). 
This may be immediately reinterpreted in terms of a collection of vector fields on $\mathcal{L}$ : we define the $n$th vector field on the affine space $\mathcal{L}$ by taking its value at $L$ to be the commutator $\left[L,\left(L_{n}\right)_{+}\right]$. A solution $L$ of the equations (3.3) of the $\mathrm{KP}$ hierarchy is then just an operator $L\left(t, t_{1}, t_{2}, \ldots\right)$ that gives (formal) integral curves of all these vector fields simultaneously. Note that the first KP time $t_{1}$ is naturally identified with translation along the original variable $t$. From the compatibility of the equations (3.3) in $x=t_{2}$ and $y=t_{3}$ (i.e. the fact that the corresponding vector fields on the space of Lax operators commute) one derives that $u=u_{1}$ satisfies the Kadomtsev-Petviashvili equation

$$
\frac{3}{4} u_{x x}=\left(u_{y}-\frac{1}{4}\left(6 u u_{t}+u_{t t t}\right)\right)_{t} .
$$

As Sato demonstrated (and we recall in the next section), the flows of the KP hierarchy (i.e. our vector fields on the space of Lax operators) may be reformulated in terms of a natural abelian Lie algebra action on (the big cell of) an infinite-dimensional Grassmannian. As a consequence, the full hierarchy (3.3) is easier to understand formally than the original KP equation (3.4) (though, as the unique solvability of the initial-value problem [Mu1] indicates, there is a close relationship between solutions $u=u(t, x, y)$ of the single equation 3.4 and solutions $L=L\left(t, t_{2}, t_{3}, \ldots\right)$ of the full KP hierarchy).

3.2. The Sato Grassmannian. For simplicity we concentrate here on the case of the usual KP hierarchy - see Section 6.1 for comments on the extension to the multicomponent KP hierarchies.

Consider the vector space $\mathcal{V}=\mathbf{C}\left(\left(\partial^{-1}\right)\right)$ of Laurent series in $\partial^{-1}$, or constant coefficient microdifferential operators. It is convenient to identify $\mathcal{V}$ with $\mathcal{E} / \mathcal{E} \cdot t$, the quotient of $\mathcal{E}$ by the left ideal generated by $t$ (i.e. the fiber of $\mathcal{E}$ at $t=0$ as a module for $\mathbf{C}[[t]]$ acting by right multiplication). The vector space $\mathcal{V}$ has a decomposition

$$
\mathcal{V}=\mathcal{V}_{+} \oplus \mathcal{V}_{-}=\mathbf{C}[\partial] \oplus \partial^{-1} \mathbf{C}\left[\left[\partial^{-1}\right]\right]
$$

Let $\operatorname{Gr}(\mathcal{V})$ denote the Sato Grassmannian: this is a space that parametrizes all subspaces $\mathcal{W} \subset \mathcal{V}$ whose projections on $\mathcal{V} / \mathcal{V}_{-}$have finite dimensional kernel and cokernel (equivalently, $\mathcal{W}$ is transversal to a subspace $\mathcal{W}^{\prime}$ which is commensurable 
with $\mathcal{V}_{-}$- one thinks of $\mathcal{W}$ as being "of the same size as $\mathcal{V}_{+}$"). ${ }^{4}$ The Sato Grassmannian has a natural structure of a scheme of infinite type (see e.g. [AMP]), and has connected components labelled by an integer, the index of a subspace $\mathcal{W} \subset \mathcal{V}$ with respect to $\mathcal{V}_{-}$. A special role is played by an open subset $\operatorname{Gr}^{\circ}(\mathcal{V})$ of the Sato Grassmannian known as the big cell: this parametrizes subspaces $\mathcal{W}$ that are transversal to $\mathcal{V}_{-}$itself, i.e.

$$
\operatorname{Gr}^{\circ}(\mathcal{V})=\left\{\mathcal{W} \in \operatorname{Gr}(\mathcal{V}): \mathcal{W} \oplus \mathbf{C}\left[\left[\partial^{-1}\right]\right]=\mathbf{C}\left(\left(\partial^{-1}\right)\right)\right\}
$$

The algebra $\mathcal{E}$ acts on $\mathcal{V}$ by left multiplication. We distinguish two pieces of the resulting symmetries of $\operatorname{Gr}(\mathcal{V})$. First, the constant coefficient microdifferential operators $\Gamma=\mathbf{C}\left(\left(\partial^{-1}\right)\right)$ act by continuous endomorphisms of $\mathcal{V}$, hence give rise to an infinite family of commuting vector fields on $\operatorname{Gr}(\mathcal{V})$. Second, consider the Volterra group

$$
\mathcal{E}_{-}^{\times}=\left\{W=1+w_{1} \partial^{-1}+w_{2} \partial^{-2}+\cdots\right\} \subset \mathcal{E}
$$

(where the $w_{i}$ are formal power series in $\left.t\right) .{ }^{5}$ The group $\mathcal{E}_{-}^{\times}$acts by continuous automorphisms on $\mathcal{V}$, hence algebraically on $\operatorname{Gr}(\mathcal{V})$; it also acts on the space $\mathcal{L}$ of Lax operators via conjugation. We will be interested in the action of the commutative subgroup

$$
\Gamma_{-}^{\times}=\mathcal{E}_{-}^{\times} \cap \Gamma=1+\partial^{-1} \mathbf{C}\left[\left[\partial^{-1}\right]\right]
$$

of constant coefficient operators (which commutes with the Lie algebra action of $\Gamma)$.

Theorem 3.1 (Sato).

(1) The action of $\mathcal{E}_{-}^{\times}$on the big cell $\operatorname{Gr}^{\circ}(\mathcal{V})$ of the index zero Grassmannian is simply transitive, i.e. every $\mathcal{W}$ has the form $\mathcal{W}=W \cdot \mathcal{V}_{+}$for a unique $W \in \mathcal{E}_{-}^{\times}$.

(2) The action of $\mathcal{E}_{-}^{\times}$on the space $\mathcal{L}$ of Lax operators $L=\partial+\cdots$ by conjugation is transitive with stabilizer $\Gamma_{-}^{\times}=1+\partial^{-1} \mathbf{C}\left[\left[\partial^{-1}\right]\right]$, i.e. every $L$ is written in the form $L=W \partial W^{-1}$ with $W \in \mathcal{E}_{-}^{\times}$unique up to constant coefficient operators.

\footnotetext{
${ }^{4}$ In terms of the natural topology of $\mathcal{V}$ giving it the structure of Tate vector space ([BD1]), $\operatorname{Gr}(\mathcal{V})$ parametrizes the $d$-lattices in $\mathcal{V}$.

${ }^{5}$ Note that the Volterra group is a pro-unipotent algebraic group with Lie algebra $\mathcal{E}_{-} \subset \mathcal{E}$ consisting of purely negative microdifferential operators.
} 
(3) The resulting isomorphism

$$
\begin{gathered}
\Gamma_{-}^{\times} \backslash \operatorname{Gr}^{\circ}(\mathcal{V}) \stackrel{\sim}{\rightarrow} \mathcal{L} \\
\text { taking } W \cdot \mathcal{V}_{+} \mapsto W \partial W^{-1} \text { identifies the infinitesimal action of } \partial^{n} \in \\
\mathbf{C}[\partial] \subset \Gamma \text { on } \mathrm{Gr}^{\circ} / \Gamma_{-}^{\times} \text {with the nth KP flow } \frac{\partial}{\partial t_{n}} \text { on Lax operators. }
\end{gathered}
$$

The operator $W \in \mathcal{E}_{-}^{\times}$associated to a subspace or Lax operator is known as the associated wave operator.

Sato's discovery of the matching of Lax operators with subspaces passes through a useful intermediary, namely a $\mathcal{D}$-module model of the big cell of $\operatorname{Gr}\left(\mathcal{V}_{+}\right)$: the big cell is identified with the space of right $\mathcal{D}$-submodules $M \subset \mathcal{E}$ that satisfy the transversality property $\mathcal{E}=M \oplus \mathcal{E}_{-}$. The identification of such $\mathcal{D}$-submodules with points of the Sato Grassmannian comes by sending $M$ to its fiber at $t=0$, i.e. the subspace $M / M \cdot t \subset \mathcal{V}=\mathcal{E} / \mathcal{E} \cdot t$ (note that this subspace no longer carries any $\mathcal{D}$-module structure in general).

A $\mathcal{D}$-submodule $M \subset \mathcal{E}$ with the transversality property $\mathcal{E}=M \oplus \mathcal{E}_{-}$is automatically a cyclic $\mathcal{D}$-module, $M \cong \mathcal{D}$. There is therefore a unique monic microdifferential operator ("wave operator") $W \in \mathcal{E}_{-}^{\times}$as above with the property that $W \cdot M=\mathcal{D}$. The Lax operator associated to $M$ is then $W \partial W^{-1}$. One of our aims in Section 4.3 will be to extend this $\mathcal{D}$-module description of the big cell to the full Grassmannian.

\section{4. $\mathcal{D}$-Bundles, Micro-Opers and Noncommutative Geometry}

4.1. Noncommutative Geometry of $\mathcal{D}$-Modules. In this section we describe the noncommutative geometry approach to $\mathcal{D}$-modules on a curve that motivates most of our constructions; see also Section 6.5 for relations with noncommutative gauge theory. Fix a curve $X$ over an algebraically closed field of characteristic zero, and let $\mathcal{D}_{X}$ denote the sheaf of differential operators on $X$. We assume for simplicity that $X$ is smooth, although a similar discussion will apply in the case of nodal or cuspidal curves if $\mathcal{D}_{X}$ is replaced by the appropriate "log" version; we refer to $[\mathrm{BN} 2]$ for more details.

To an algebraic variety one may associate an abelian category, namely its category of (quasi)coherent sheaves, that encodes the fundamental geometry of the 
variety. In noncommutative algebraic geometry one takes the abelian category ${ }^{6}$ itself as the starting point - see $[\mathrm{St}, \mathrm{StV}]$ for an introduction to this point of view. For example, a fundamental construction of noncommutative algebraic geometry associates, to a sheaf of noncommutative algebras $\mathcal{A}$ on a variety, the category of $\mathcal{A}$-modules.

Starting with the sheaf $\mathcal{D}_{X}$ of differential operators on a smooth curve this construction defines a noncommutative variety, the category of (quasicoherent) $\mathcal{D}_{X^{-}}$ modules. The algebra $\mathcal{D}_{X}$ is a deformation of the commutative algebra $\operatorname{Sym} \bullet\left(T_{X}\right)$, the sheaf of symbols of differential operators. Since the latter is the pushforward to $X$ of the algebra of functions on the cotangent bundle $T^{*} X$ of $X$, one thinks of $\mathcal{D}_{X}$ as the algebra of functions on a "quantization of the cotangent bundle of $X$ ", a noncommutative algebraic surface that we will denote by $\operatorname{Spec}(\mathcal{D})=T_{\hbar}^{*} X$. It turns out that the intuition provided by thinking of the category of $\mathcal{D}_{X}$-modules in this way is an excellent guide to many interesting questions and useful constructions concerning $\mathcal{D}$-modules.

4.1.1. Completion. In studying moduli problems for sheaves, one prefers to work with a projective (or proper) variety. In particular, moduli problems of torsionfree sheaves on the open surface $T^{*} X$ or on its deformation $T_{\hbar}^{*} X$ (i.e. of $\mathcal{D}$ bundles) will not be well behaved, even if we assume (as we do in the rest of the section) that the curve $X$ is projective. Thus it is convenient to compactify to a proper variety by adding a divisor $X_{\infty}$ at infinity and then consider framed sheaves, namely sheaves that are trivialized at infinity. Such framing conditions can be considered as "asymptotic decay conditions at infinity." Indeed, framing conditions arise naturally in Yang-Mills theory when one studies connections on a noncompact 4-manifold that have suitable decay conditions at infinity on their curvature: such connections often admit extensions to connections on the complex projective surface that automatically are trivialized at infinity.

In the case of the vector bundle $T^{*} X$ there is a standard completion to a projective bundle $T^{*} X \subset \overline{T^{*} X}=\mathbb{P}\left(T^{*} X \oplus \mathcal{O}\right) \supset X_{\infty}$, by adding the curve $X_{\infty}=\mathbb{P}\left(T^{*} X\right) \cong X$ itself at infinity. Thus we consider the projective bundle with homogeneous coordinate rings $S^{\bullet}=\operatorname{Sym}^{\bullet}\left(T_{X} \oplus \mathcal{O}_{X}\right)=\oplus_{k}\left(\operatorname{Sym}^{\leq k} T_{X}\right) \cdot t^{k}$.

\footnotetext{
${ }^{6}$ In fact, it may be better to replace the category of quasicoherent sheaves on a variety by its derived category as a differential graded category, [Dr], and take DG categories as the starting point.
} 
The category of coherent sheaves on $\overline{T^{*} X}=\underline{\operatorname{Proj}}\left(S^{\bullet}\right)$ is equivalent to the quotient of the category of finitely generated graded $S^{\bullet}$-modules modulo its subcategory of bounded modules. Furthermore, restriction of a coherent sheaf $\widetilde{M}$ on $\overline{T^{*} X}$ to the curve $X_{\infty}=\underline{\operatorname{Proj}}\left(\mathrm{Sym}^{\bullet} T_{X}\right)$ at infinity is given by taking the graded $S^{\bullet}$-module $M$ to the graded $\operatorname{Sym}^{\bullet}\left(T_{X}\right)$-module $M / t M$.

The natural quantization of the algebra $S^{\bullet}$, and hence of the projective completion $\overline{T^{*} X}$ of $T^{*} X$, is given by the Rees ring $\mathcal{R}\left(\mathcal{D}_{X}\right)=\oplus_{k} \mathcal{D}_{X}^{k} \cdot t^{k}$ of $\mathcal{D}_{X}$, where $\mathcal{D}_{X}^{k}$ denotes differential operators of order at most $k$. A graded module over the Rees ring gives a filtered module over the filtered ring $\mathcal{D}_{X}$ and conversely. Since we are interested in such modules modulo bounded modules, we should consider $\mathcal{D}$ modules which are eventually filtered, in other words only sufficiently high filtered pieces are defined (more precisely, morphisms are only required to respect the filtration eventually). This leads us to consider the (derived) category of eventually filtered $\mathcal{D}$-modules as the completion $\overline{T_{\hbar}^{*} X}$ of the noncommutative variety $T_{\hbar}^{*} X$. The associated graded ring of $\mathcal{D}$ is just the commutative $\operatorname{ring} \operatorname{gr}(\mathcal{D})=\operatorname{Sym} \bullet T_{X}$. It follows that our noncommutative variety is described by adjoining to $T_{\hbar}^{*} X$ the commutative curve $X_{\infty}=\operatorname{Proj}(\operatorname{gr}(\mathcal{D})) \cong X$. The restriction of an eventually filtered $\mathcal{D}$-module $M$ to $X_{\infty}$ is determined by its asymptotics, i.e. by the coherent sheaf $\operatorname{gr}_{N} M$ for $N \gg 0$.

4.1.2. The noncommutative variety $\overline{T_{\hbar}^{*} X}$ has the same $K$-group and deRham cohomology (i.e. cyclic homology) as the variety $\overline{T^{*} X}$ of which it is a deformation. (In fact Laumon [La1] shows that $T_{\hbar}^{*} X$, i.e. the derived category of $\mathcal{D}$-modules, has the same $K$-group as well). Therefore filtered $\mathcal{D}$-modules have the same numerical invariants as coherent sheaves on the completed cotangent bundle. In particular it makes sense to speak of the Chern classes of a filtered $\mathcal{D}$-bundle, and to try to deform moduli spaces of sheaves with fixed numerical invariants (such as Hilbert schemes of points) from $\overline{T^{*} X}$ to $\overline{T_{\hbar}^{*} X}$.

It is interesting to note that the "surface" $T_{\hbar}^{*} X$ exhibits both one and twodimensional features, accounting for some of the peculiarities of noncommutative instantons. In particular, it has (cohomological) dimension one, and every torsion-free sheaf on it ( $\mathcal{D}_{X}$-module) is projective, and so may be considered a vector bundle. However these "bundles" are similar to torsion-free sheaves in the commutative limit, and their moduli provide (flat) deformations of the moduli spaces of the latter. In particular they carry a "second Chern class" (or instanton 
number) $c_{2}(M)$, which may be defined cohomologically, algebraically (using the filtration), or geometrically (using the associated graded sheaf on $T^{*} X$ ).

4.1.3. Microlocalization. In order to interpret the microdifferential Lax operators of the KP hierarchy geometrically, we will use the interpretation of microlocalization in terms of the noncommutative space $\overline{T_{\hbar}^{*} X}$ - for more on microlocalization, see for example the books [Ka, Sh] and the papers [Sp, EO, AVV]. Recall that the sheaf $\mathcal{D}$ embeds in a sheaf of algebras $\mathcal{E}$, the sheaf of microdifferential operators, defined as follows. First we adjoin to $\mathcal{D}$ (in local coordinates) the formal inverse $\partial^{-1}$ of a nonvanishing vector field $\partial$ satisfying the Leibniz rule (3.2), and then we complete with respect to powers of $\partial^{-1}$, so that in local coordinates $\mathcal{E}$ is given by (noncommutative) Laurent series in $\partial^{-1}$ over $\mathcal{O}_{X}$. The sheaf $\mathcal{E}$ is $\mathbf{Z}$-filtered by order in $\partial$, extending the filtration on $\mathcal{D}$, and the completed associated graded algebra is the commutative algebra given in local coordinates by $\mathcal{O}_{X}\left(\left(\xi^{-1}\right)\right)$, where $\xi$ is the symbol of $\partial$. The subsheaf of microdifferential operators of degree at most zero forms a subalgebra $\mathcal{E}_{-} \subset \mathcal{E}$ which is complete with respect to the natural topology.

The geometric interpretation of $\mathcal{E}$ is clear once we note that the completed associated graded algebra of $\mathcal{E}$ is naturally identified with Laurent series along the section at infinity $X_{\infty} \subset \overline{T^{*} X}$ of the compactified cotangent bundle, while the associated graded of the subalgebra $\mathcal{E}_{-}$consists of Taylor series along the same section. Informally, while $\partial$ plays the role of coordinate "along the fibers" on $T_{\hbar}^{*} X$, its inverse $\partial^{-1}$ plays the role of coordinate near the section $X_{\infty}$ at infinity, so we may consider $\mathcal{E}_{-}$as functions in the formal neighborhood of $X_{\infty} \subset \overline{T_{\hbar}^{*} X}$ and $\mathcal{E}$ as Laurent series along this section. ${ }^{7}$ In particular, for a (right) $\mathcal{D}$-module $M$ (sheaf on $T_{\hbar}^{*} X$ ) we have an associated $\mathcal{E}$-module $M_{\mathcal{E}}=M \otimes_{\mathcal{D}} \mathcal{E}$, which is the restriction of $M$ to the punctured formal neighborhood of $X_{\infty}$, while for a filtered $\mathcal{D}$-module (sheaf on $\overline{T_{\hbar}^{*} X}$ ) we have an associated $\mathcal{E}_{-}$-module $M_{-}=\left(M_{\mathcal{E}}\right)_{\leq 0}$, the piece of nonpositive filtration with respect to the induced filtration of $M_{\mathcal{E}}$, which is the restriction of $M$ to the formal neighborhood of $X_{\infty}$.

Remark 4.1 (Higher-Dimensional Microlocalization). For a smooth $n$-dimensional variety $X$, microdifferential operators $\mathcal{E}$ form a sheaf on the projectivized cotangent bundle $X_{\infty}:=\mathbb{P}\left(T^{*} X\right)$, which is isomorphic to $X$ for $n=1$. Here again $X_{\infty}$ appears as the divisor at infinity in the projective completion of $T^{*} X$ or of its

\footnotetext{
${ }^{7}$ See $[$ PRo] for a discussion in the context of Kapranov's formal NC schemes.
} 
noncommutative deformation $T_{\hbar}^{*} X$ on which $\mathcal{D}$-modules live, and microdifferential operators are again formal Laurent series along this divisor in the quantized cotangent bundle.

4.2. $\mathcal{D}$-Bundles. In this section we introduce $\mathcal{D}$-bundles on a curve.

Definition 4.2. [BD2] A $\mathcal{D}$-bundle $M$ on $X$ is a locally projective, coherent right $\mathcal{D}_{X}$-module.

On a smooth (or more generally cuspidal) curve $X$, any torsion-free $\mathcal{D}_{X}$-module is locally projective. However a general rank $1 \mathcal{D}$-bundle $M$ on a curve $X$ is not locally free, but only generically free: away from finitely many points of $X, M$ is isomorphic to $\mathcal{D} .^{8}$

Example 4.3 (Right ideals in $\mathcal{D}_{X}$ ). Every right ideal in $\mathcal{D}_{X}$ is torsion-free, hence a $\mathcal{D}$-bundle of rank 1 . However, ideals in $\mathcal{D}_{X}$ are typically not locally free. Consider for example $X=\mathbf{A}^{1}$, so that $\mathcal{D}_{X}$ is the Weyl algebra $\mathbf{C}\langle z, \partial\rangle /\{\partial z-z \partial=$ $1\}$. The right ideal generated by $z^{2}$ and $1-z \partial$ is not locally free near $z=0$. However, a right ideal of $\mathcal{D}_{X}$ is generically locally free over $\mathcal{D}_{X}$, and in fact is equivalent (under rescalings by $\mathcal{D}_{X}$ ) to a right ideal in $\mathcal{D}_{X}$ which agrees with $\mathcal{D}_{X}$ generically. Following [CH1] (see also [BD2, BW2, BN1]), such an ideal is determined by the (finite) collection of points $x$ at which it differs from $\mathcal{D}_{X}$ and choices of subspaces of $\widehat{\mathcal{O}}_{x}$ at those points $x$; for example, the right ideal above corresponds to the subspace $\mathbf{C}+\left(z^{2}\right)$ in $\mathbf{C}[[z]]$. (These collections of subspaces form the adèlic Grassmannian, see Section 4.5.)

If $M$ is a rank $1 \mathcal{D}$-bundle on $X$, we refer to the finite subset $S$ of $X$ consisting of points $s \in S$ such that $M$ is not isomorphic to $\mathcal{D}$ in any neighborhood of $s$, as the set of cusps of $M$. The terminology is motivated by the description of $\mathcal{D}$-modules developed in [CH2], and amplified and generalized in [BN1], in terms of coherent sheaves on singular varieties.

In order to construct moduli spaces for $\mathcal{D}$-bundles on $X$, it is important to "compactify" the surface $T_{\hbar}^{*} X$ to the noncommutative $\mathbf{P}^{1}$-bundle $\overline{T_{\hbar}^{*} X}$ as explained in Section 4.1, in other words to consider filtered $\mathcal{D}$-bundles (we require the graded components to be vector bundles on $X$ ). Moreover, we would like to

\footnotetext{
${ }^{8}$ Since $\mathcal{D}$ has a skew field of fractions it follows that locally projective $\mathcal{D}$-modules have welldefined ranks.
} 
fix the geometry of the $\mathcal{D}$-bundle along the (commutative) curve $X_{\infty}$ at infinity in $\overline{T_{\hbar}^{*} X}$, in other words to consider framed $\mathcal{D}$-bundles:

Definition 4.4 (See Definition 3.2 of [BN2]).

(1) For a filtered $\mathcal{D}$-module $M$, we denote by $\left.M\right|_{X_{\infty}}$ the coherent sheaf on $X_{\infty}=\underline{\operatorname{Proj}}(\operatorname{gr}(\mathcal{D}))$ associated to the graded $\operatorname{gr}(\mathcal{D})$-module $\operatorname{gr}(M)$.

(2) A (trivially) framed $\mathcal{D}$-bundle is a filtered $\mathcal{D}$-bundle $M$ equipped with an isomorphism $\left.M\right|_{X_{\infty}} \rightarrow \mathcal{O}_{X_{\infty}}$.

We let $\mathfrak{M}_{n}\left(\overline{T_{\hbar}^{*} X}\right)$ denote the moduli stack of framed $\mathcal{D}$-bundles of second Chern class $n$. The precise definition of the moduli stack $\mathfrak{M}_{n}\left(\overline{T_{\hbar}^{*} X}\right)$ (as well as its generalization to other framings) appears in [BN2]; for a projective curve $X, \mathfrak{M}_{n}\left(\overline{T_{\hbar}^{*} X}\right)$ is an algebraic stack, and in the case of an elliptic curve will be described explicitly using the Fourier-Mukai transform in Section 5.

4.3. Micro-Opers and Lax Operators. In this section we introduce enhanced versions of $\mathcal{D}$-bundles, the micro-opers, which give a geometric form to the Lax operators of the KP hierarchy. On a (commutative) variety, functions act by $\mathcal{O}$-module endomorphisms of any $\mathcal{O}$-module. Analogously, we will need a slight enhancement of the structure of framed $\mathcal{D}$-bundle, giving the action of a commutative subalgebra of "functions" as endomorphisms. Throughout this section, $(X, \partial)$ will denote a curve with a fixed nowhere-vanishing vector field (so that $X$ is an elliptic curve or affine).

Definition 4.5. A micro-oper structure on an $\mathcal{O}$-framed $\mathcal{D}$-bundle $M$ is the data of an $\mathcal{E}$-module endomorphism $\partial_{M}$ of $M_{\mathcal{E}}=M \otimes_{\mathcal{D}} \mathcal{E}$, with principal symbol $\partial$ with respect to the induced filtration of $M_{\mathcal{E}}$.

Remark 4.6. See Section 6.1 and [BN2] for discussion of the higher-rank version of multi-opers, which will give (matrix) Lax operators for multicomponent KP hierarchies.

The endomorphism $\partial_{M}$ commutes with the right action of $\mathcal{E}$, and is required to perturb the degree of the filtration by one, $\partial_{M}:\left(M_{\mathcal{E}}\right)_{i} \rightarrow\left(M_{\mathcal{E}}\right)_{i+1}$, inducing an isomorphism $\operatorname{gr}\left(\partial_{M}\right): \operatorname{gr}_{n} M_{\mathcal{E}} \rightarrow \operatorname{gr}_{n+1} M_{\mathcal{E}}$ on the graded pieces compatible with the framing. Thus a micro-oper structure consists of a lifting of the vector 
field $\partial$ to $M_{\mathcal{E}}$, which we may think of as (part of) a connection ${ }^{9}$ that satisfies a strict form of Griffiths transversality.

We see that micro-opers are closely analogous to the opers introduced by Beilinson and Drinfeld [BD1] following Drinfeld and Sokolov [DS]. A $G L_{n}$-oper on $X$ is a rank $n$ bundle $V$ with a full flag $\left\{V_{i}\right\}$ and a connection, which satisfies strict Griffiths transversality: $\nabla_{\partial}: V_{i} \rightarrow V_{i+1}$, and induces an isomorphism $V_{i} / V_{i-1} \rightarrow V_{i+1} / V_{i}$. It is easy to see that $G L_{n}$-opers are naturally identified with monic $n$th order differential operators acting between line bundles on the curve $X$. Thus $G L_{n}$ opers give a coordinate-free form of the Lax operators of the $n$th $\mathrm{KdV}$ hierarchy, which is amenable to generalizations to arbitrary reductive groups. (See Section 4.4 for more on the parallels of micro-opers with opers.)

The importance of micro-opers comes from the following theorem, extending Sato's description of the KP flows on Lax operators on the disc to micro-opers on a curve $(X, \partial)$. Let $\Gamma_{-}^{\times}$denote the multiplicative group $1+\partial^{-1} \mathbf{C}\left[\left[\partial^{-1}\right]\right] \subset \mathcal{E}(X)$ and $\Gamma_{+}=\mathbf{C}[\partial]$ considered as an abelian Lie algebra. For $x \in X$, we let $\mathcal{E}_{x}$ denote the fiber of $\mathcal{E}$ at $x$ with respect to the right $\mathcal{O}$-module structure, i.e. $\mathcal{E}_{x}=\mathcal{E} / \mathcal{E} \cdot \mathfrak{m}_{x}{ }^{10}$

Theorem 4.7 (Section 3.5 of $[\mathrm{BN} 2]$ ).

(1) The quotient $\Gamma_{-}^{\times} \backslash \operatorname{Gr}\left(\mathcal{E}_{x}\right)$ of the Sato Grassmannian is in bijection with micro-opers on the formal disc at $x$.

(2) For any $x \in X$, we have a canonical embedding of the space $\mathcal{M O} p(X)$ of micro-opers on $X$ into the quotient of the Sato Grassmannian $\Gamma_{-}^{\times} \backslash \operatorname{Gr}\left(\mathcal{E}_{x}\right)$, preserved by the KP flows (given by the action of $\Gamma_{+}$on $\Gamma_{-}^{\times} \backslash \operatorname{Gr}\left(\mathcal{E}_{x}\right)$ ).

(3) The $\mathcal{D}$-bundle $M$ underlying a micro-oper is locally free near $x$ if and only if the image of $M$ in $\Gamma_{-}^{\times} \backslash \operatorname{Gr}\left(\mathcal{E}_{x}\right)$ is in the image of the big cell.

It follows that a micro-oper on $X$ defines (and is determined by) a canonical KP Lax operator on the dense open subset $U \subset X$ on which the associated point of the Grassmannian is in the big cell, i.e. where the $\mathcal{D}$-bundle is locally free. In this sense the structure of micro-oper gives precise meaning to the singularities

\footnotetext{
${ }^{9}$ To be more precise, we may think of the action of $\partial$ as a part of an additional left action of $\mathcal{D}$ on $M_{\mathcal{E}}$.

${ }^{10}$ This vector space is isomorphic to the vector space $\mathcal{V}=\mathbf{C}\left(\left(\partial^{-1}\right)\right)$ from Section 3.2 using any local coordinate at $x$, in particular the coordinate coming from the vector field $\partial$ near $x$.
} 
of a microdifferential operator. Thus the space $\mathcal{M O} p(X)$ provides the natural completed phase space for solutions of the KP hierarchy whose dependence on the first KP time is meromorphic on $X$.

4.3.1. Trivialized Micro-Opers. The proof of Theorem 4.7 breaks down into two parts: relating the full Sato Grassmannian with a parameter space of $\mathcal{D}$-modules, the trivialized micro-opers, and reducing a micro-oper to an "abelian gauge".

Let $U \subset X$ denote an open subset.

Definition 4.8. A trivialization of a micro-oper $M$ on $U$ is the data of an isomorphism $\widetilde{\eta}: M \otimes_{\mathcal{D}} \mathcal{E} \rightarrow \mathcal{E}$ on $X$, or equivalently of a full embedding $\eta: M \hookrightarrow \mathcal{E}$ (i.e. an embedding inducing such an isomorphism), compatible with the framing on $M$.

Note that a micro-oper $M$ determines an $\mathcal{E}_{-}$-module $M_{-}$, the zeroth filtered piece of the filtered $\mathcal{E}$-module $M_{\mathcal{E}}=M \otimes_{\mathcal{D}} \mathcal{E}$. A trivialization of a micro-oper can equivalently be described as an isomorphism of $\mathcal{E}_{-}$-modules $M_{-} \rightarrow \mathcal{E}_{-}$. From the noncommutative geometry point of view, a trivialization of a micro-oper $M$ is a formal framing, i.e. an extension of the trivialization (framing) of $M$ on the section $X_{\infty}$ at infinity to its formal neighborhood. Such extensions form a torsor for the pro-unipotent Volterra group $\mathcal{E}_{-}^{\times}$on $X$ and can be shown to exist on any affine open $U$.

Let $x \in X$. To a trivialized micro-oper $M \hookrightarrow \mathcal{E}$ on $X$ we assign its fiber $M_{x}=M /\left(M \cdot \mathfrak{m}_{x}\right) \rightarrow \mathcal{E}_{x}=\mathcal{E} / \mathcal{E} \cdot \mathfrak{m}_{x}$. One checks that $M_{x}$ is a $d$-lattice in the vector space $\mathcal{E}_{x}$, i.e. a point of the Sato Grassmannian $\operatorname{Gr}\left(\mathcal{E}_{x}\right)$. This defines a map from trivialized micro-opers to the Sato Grassmannian. On the formal disc, we show that this map is an isomorphism of moduli functors between trivialized micro-opers and the full Sato Grassmannian. When the $\mathcal{D}$-module $M \hookrightarrow \mathcal{E}$ is cyclic, we recover Sato's description of the big cell Gr ${ }^{\circ}$. This provides the analog of Theorem 4.7 for trivialized micro-opers.

It follows from Sato's identification of the big cell with jets of microdifferential operators that a trivialized micro-oper $(M, \eta)$ on $U$ defines a canonical element of the Volterra group, the wave operator $W_{M} \in \mathcal{E}_{-}^{\times}\left(U^{\prime}\right)$, on the open subset $U^{\prime} \subset U$ where $M$ is locally free. We may use the vector field $\partial$ to define a formal coordinate on the formal disc $\widehat{D}_{x}$ at $x$. This coordinate induces an isomorphism 
from the Sato Grassmannian $\mathrm{Gr}=\operatorname{Gr}(\mathcal{V})$ to the Sato Grassmannian at $x, \operatorname{Gr}\left(\mathcal{E}_{x}\right)$, and hence to trivialized micro-opers on $\widehat{D}_{x}$. Thus any trivialized micro-oper on $X$ defines by restriction to $\widehat{D}_{x}$ a point of Gr.

The algebra $\mathcal{E}(X)$ acts on the left of $\mathcal{E}$ by endomorphisms of $\mathcal{E}$ as a right $\mathcal{E}$-module and hence on the collection of right $\mathcal{D}$-submodules of $\mathcal{E}$. Thus $\mathcal{E}(X)$, considered as a Lie algebra, acts on trivialized micro-opers on $X$. It is then automatic from Sato's description of the KP flows as the action of $\mathbf{C}[\partial]$ on $\mathrm{Gr}$ that the action of the vector field $\partial_{n}$ restricts to the action of $\partial^{n} \in \mathbf{C}[\partial]$ on the image of $\mathcal{M O}_{p}(X)$ in Gr. Note that the first KP time, the action of $\partial$ itself, simply translates infinitesimally along $X$.

4.3.2. Gauging Micro-Opers. To complete the proof of Theorem 4.7, we show that every micro-oper has a canonical $\Gamma_{-}^{\times}$-orbit of local coordinatizations on $M$, namely the trivializations in which the action of $\partial_{M}$ on $M_{\mathcal{E}}$ is identified with the left action of $\partial$ on $\mathcal{E}$. To see this pick any filtered local trivialization $\widetilde{\eta}: M_{\mathcal{E}} \rightarrow \mathcal{E}$, and note that since $\partial_{M}$ is acting by right $\mathcal{E}$-module endomorphisms of $M_{\mathcal{E}}, \widetilde{\eta}\left(\partial_{M}\right)$ must act on $\mathcal{E}$ by left multiplication by an operator of the form $\partial+a_{0}+a_{1} \partial^{-1}+\cdots$. Changing the trivialization by the left action of $\mathcal{E}_{-}^{\times}$on $\mathcal{E}$, we can conjugate $\widetilde{\eta}\left(\partial_{M}\right)$ to $\partial$, and do so uniquely up to the centralizer of $\partial$ in $\mathcal{E}_{-}^{\times}$, namely $\Gamma_{-}^{\times}$. This defines the desired $\Gamma_{-}^{\times}$-orbit of trivialized micro-oper, which is the inverse to the forgetful map from trivialized micro-opers. The rest of Theorem 4.7 follows easily.

4.4. KdV and Affine Opers. The point of view on micro-opers and the main construction in Theorem 4.7 are closely parallel to the interpretation in $[\mathrm{BF}]$ of the Drinfeld-Sokolov generalized KdV hierarchies [DS]. Namely the microopers are the KP analogues of the loop group bundles with connection called affine opers in $[\mathrm{BF}]$. We provide a quick overview of the description of the KdV hierarchies in $[\mathrm{BF}]$, which will not be needed in what follows ${ }^{11}$. We then show how micro-opers satisfying an n-periodicity condition are naturally identified with $G L_{n}$ affine opers.

Recall that $G L_{n}$-affine opers on a curve $X$ are quadruples $\left(V, \nabla, V_{\infty}\right)$ consisting of a vector bundle $V$ on $X \times \mathbf{P}^{1}$, a connection $\nabla$ along $X$ on the bundle of sections $V_{D^{\times}}$of $V$ on the punctured disc $D^{\times}$at $\infty$ times $X$, and a flag $V_{\infty}^{\cdot}$ on the fiber $V_{\infty}$

\footnotetext{
${ }^{11}$ We restrict our attention to the $n$th $\mathrm{KdV}$ hierarchy, associated to $G L_{n}$; the construction of $[\mathrm{BF}]$ applies to the Drinfeld-Sokolov hierarchies associated to any semisimple group.
} 
of $V$ at $X \times \infty$, satisfying two conditions. First, $\nabla$ preserves the subbundle $V_{\mathbf{A}}^{1}$ of regular sections of $V$ on $\mathbf{A}^{1}$. Second, $\nabla$ satisfies strict Griffiths transversality (i.e. the oper condition) with respect to the filtration $V_{D^{\times}}$on $V_{D^{\times}}$refining the filtration by order of pole at infinity using the flag $V_{\infty}$. Namely the connection perturbs the filtration by one,

$$
\nabla_{\partial}: V_{D^{\times}}^{i} \rightarrow V_{D^{\times}}^{i+1}
$$

and induces isomorphisms on graded pieces

$$
\nabla_{\partial}: V_{D^{\times}}^{i} / V_{D^{\times}}^{i-1} \simeq V_{D^{\times}}^{i+1} / V_{D^{\times}}^{i}
$$

Every affine oper has a canonical reduction - the Drinfeld-Sokolov gauge - to an abelian (Heisenberg) subgroup $A$ of the loop group. In this gauge there is an evident action of the Heisenberg group on the space of affine opers is evident, and this action defines the $\mathrm{KdV}$ hierarchy of commuting flows. It is proven in $[\mathrm{BF}]$ that affine opers on the disc are parametrized by the quotient $\mathbf{G r}_{n} / A_{<}$ of the $G L_{n}$ (thick) loop Grassmannian $\mathbf{G r}_{n}=G L_{n}\left(\mathbf{C}\left(\left(z^{-1}\right)\right)\right) / G L_{n}(\mathbf{C}[z])$ by the positive half $A_{<}{ }^{12}$ of $A$. Generic affine opers, the image $\mathbf{G r}_{n}^{\circ} / A_{<}$of the big cell in the above Grassmannian, correspond to triples $\left(V, \nabla, V^{\cdot}\right)$ with $V$ a trivial vector bundle. The generic affine opers are then identified with $\mathrm{KdV}$ Lax operators, i.e. $n$th order differential operators with power series coefficients $L=\partial^{n}+u_{1} \partial^{n-1}+\cdots+u_{n} \in \mathcal{D}$. The description as a quotient of the Grassmannian by a torus in the loop group results in an identification of the KdV phase space with a space of vector bundles equipped with germs of Higgs fields, or equivalently germs of spectral curves. This gives an algebraic approach to the association of spectral curves (which are formal branched covers of $\mathbf{P}^{1}$ ) to these Lax operators, and establishes a natural bijection between the moduli space of spectral data and that of differential operators.

Proposition 4.9. There is a natural identification between affine $G L_{n}$-opers on $X$ and micro-opers $\left(M, \partial_{M}\right)$ such that $\partial_{M}^{n}$ preserves the submodule $M \subset M_{\mathcal{E}}$. The identification preserves big cells: $M$ is locally free if and only if the corresponding affine oper is generic.

Proof. To a micro-oper we assign an affine oper as follows. Let $V_{D^{\times}}=M_{\mathcal{E}}^{l}=$ $M_{\mathcal{E}} \otimes \omega_{X}^{-1}$, considered as an $\mathcal{O}_{X}\left(\left(z^{-1}\right)\right)$-module via the endomorphism $z=\partial_{M}^{n}$.

\footnotetext{
${ }^{12} A_{+}$in the notation of $[\mathrm{BF}]$.
} 
The right $\mathcal{D}$-module structure on $M_{\mathcal{E}}$ defines the desired connection $\nabla$ on $V_{D^{\times}}$. The vector bundle $V$ is defined by gluing the $\mathcal{O}_{X}[z]$-module $V_{\mathbf{A}}^{1}=M^{l}$ and the $\mathcal{O}_{X}\left[\left[z^{-1}\right]\right]$-module $V_{D}=M_{<}^{l}$ (again with $z$ acting as $\partial_{M}^{n}$ ) using the identifications

$$
M^{l} \otimes_{\mathcal{O}_{X}[z]} \mathcal{O}_{X}\left(\left(z^{-1}\right)\right)=M_{\mathcal{E}}^{l}=M_{<}^{l} \otimes_{\mathcal{O}_{X}\left[\left[z^{-1}\right]\right]} \mathcal{O}_{X}\left(\left(z^{-1}\right)\right)
$$

The flag $V_{\infty}$ is induced by the filtration on $M_{<}$. The $\mathcal{D}$-module structure on $V_{D^{\times}}$ preserves the $\mathcal{D}$-submodule $V_{\mathrm{A}}^{1}=M^{l}$, and perturbs the good filtration on $V_{D^{\times}}$ as required by the very definition of a good filtration.

Conversely, reversing the above assignments we obtain the right $\mathcal{D}$-module $M_{\mathcal{E}}=V_{D^{\times}} \otimes \omega_{X}$, which is automatically an $\mathcal{E}$-module since the connection operator is invertible. The rest of the structure of micro-oper on $M=V_{\mathbf{A}}^{1} \otimes \omega_{X}$ follows easily.

It is easy to see that the big cell condition for $M$ matches precisely the triviality of the vector bundle $V$ on $\mathbf{P}^{1}$, i.e. the transversality of $V_{\mathbf{A}}^{1}$ and $z^{-1} V_{D}$.

4.5. Micro-Opers and the Adèlic Grassmannian. Micro-opers also give rise to solutions of the KP hierarchy by a completely different route, that is closely related to the description of ideals in the Weyl algebra and more general projective $\mathcal{D}$-modules developed in [CH1, CH2, BW1, BGK2] (see [BN1] for a different approach, closer in spirit to the current work, as well as generalizations to higher dimensions).

It is easy to check (see [BN2]) that a framed $\mathcal{D}$-bundle on $X$ carries a canonical trivialization (identification with $\mathcal{D}$ ) on the open set where it is smooth (i.e. the local data lie in the big cell). Note that the existence of this canonical generic trivialization is to be expected from the point of view of bundles on a ruled surface. If $S \rightarrow X$ is a (commutative) ruled surface with section $X_{\infty} \subset S$, then a framed vector bundle on $S$ has a well-defined generic splitting type. If this generic type is trivial, then the additional data of a framing determines a unique trivialization of the bundle away from finitely many jumping fibers.

The isomorphism classes of $\mathcal{D}$-bundles on a curve $X$ equipped with a generic trivialization, i.e. embedding as an ideal in rational differential operators $\mathcal{D}(\mathbf{C}(X))$, are in natural bijection, via the deRham functor $M \mapsto M \otimes_{\mathcal{D}} \mathcal{O}$, with the adèlic 
Grassmannian $\mathfrak{G r}^{a d}(X)$ of Wilson [W3]. ${ }^{13}$ The latter parametrizes certain subspaces of the rational functions $\mathbf{C}(X)$ on $X$, defined by independent conditions at finitely many points of $X$. Equivalently, $\mathfrak{G r}^{a d}(X)$ parametrizes torsion-free sheaves of rank one (with generic trivialization) on cuspidal quotients of $X$, singular curves having $X$ as their bijective normalization. (The $\mathcal{D}$-module is obtained from the torsion-free sheaf by a variant of the induction functor from $\mathcal{O}$-modules on $X$ to $\mathcal{D}$-modules, $M \mapsto M \otimes_{\mathcal{O}} \mathcal{D}$.)

In this way we can see that $\mathfrak{G r}^{a d}(X)$ appears as a phase space for algebrogeometric solutions (à la Krichever) of the KP hierarchy, attached to all cuspidal quotients of $X$. Namely, to a rank one torsion-free sheaf on a curve, equipped with a trivialization near a smooth point $\infty$, one assigns its vector space of sections away from $\infty$, which (using the trivialization and a local coordinate $z^{-1}$ ) define a subspace of $\mathbf{C}\left(\left(z^{-1}\right)\right)$, i.e. a point in the Sato Grassmannian $\operatorname{Gr}\left(\mathbf{C}\left(\left(z^{-1}\right)\right)\right)$.

Thus we have a construction of algebro-geometric solutions of KP from microopers on $X$ (which depends only on the underlying $\mathcal{D}$-bundle). Note that here $X$ and its cuspidal quotients are playing the role of the spectral curve of KP, while in our construction in Theorem 4.7 the curve $X$ corresponds to the first time of KP, i.e. the (usually formal) curve where Lax operators live. For a curve of genus greater than one, there is no intersection between these spaces of "algebraic" (Krichever) and "differential" (micro-oper) solutions to KP. In the rational, trigonometric and elliptic cases, it follows from Theorem 5.8 that all differential solutions (i.e. micro-opers on cubic curves) are in fact algebro-geometric solutions, assigned to tangential covers. In the rational case, furthermore, there is a symmetry of spectral and differential variables, namely the Fourier transform, giving rise to the bispectral involution of Wilson [W1] identifying the two classes of solutions - see Section 6.2.

\section{Fourier Duality}

5.1. The Fourier-Mukai Transform. We recall the Fourier-Mukai transform for abelian varieties, in the special case of an elliptic curve $E$ :

Theorem 5.1. Let $E$ denote an elliptic curve and $\mathcal{P}$ the Poincaré sheaf on $E \times E$.

\footnotetext{
${ }^{13}$ More precisely, one obtains the adèlic Grassmannian by also requiring that $M \otimes_{\mathcal{D}} \mathcal{O}$ has index 0 in $\mathbf{C}(X)$ at every point.
} 
(1) (Mukai $[\mathrm{Muk}])$ The functor $\mathbb{F}: M \mapsto R p_{2 *}\left(p_{1}^{*} M \otimes \mathcal{P}\right)$ defines an autoequivalence of the bounded derived category of coherent sheaves on $E$ (and likewise for quasicoherent sheaves).

(2) (Laumon [La2], Rothstein [Ro2]) $\mathbb{F}$ induces an equivalence $\mathbb{F}: D^{b}\left(\mathcal{D}_{E}\right) \rightarrow$ $D^{b}\left(E^{\natural}\right)$ of bounded derived category of coherent $\mathcal{D}$-modules on $E$ and of coherent sheaves on $E^{\natural}$. ${ }^{14}$

Morally, $\mathbb{F}$ describes a coherent sheaf on $E$ as a "direct integral" of degree zero line bundles, which are parametrized by points of the dual abelian variety, $E$ itself. The extension in the second part writes a $\mathcal{D}$-module on $E$ as a "direct integral" of flat bundles on $E$, which are parametrized by points of $E^{\natural}$. From the noncommutative geometry point of view, the first part defines an automorphism of the "noncommutative variety" $D^{b}(E)$ defined by $E$, while the second identifies the noncommutative variety $T_{\hbar}^{*} E$ with that underlying the commutative variety $E^{\natural}$.

In [BN2] we extend the Fourier-Mukai transform to arbitrary Weierstrass cubics; see $[\mathrm{FM}]$ for the case of semistable bundles of degree zero. We also generalize the Fourier-Mukai transform for $\mathcal{D}$-modules to the singular setting, utilizing results of $[\mathrm{PRo}]$ on Fourier transforms for $D$-algebras. More precisely, we consider the sheaf $\mathcal{D}^{\log }$ of log-differential operators with respect to the singularity of the cubic: this is the subsheaf of all differential operators generated by $\mathcal{O}_{E}$ and the translation-invariant vector fields coming from the action of the group $\mathbb{G}$ on $E$. We introduce the surface $E^{\natural} \rightarrow E$ as the affine bundle classifying rank one

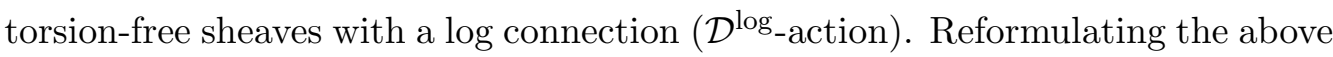
theorems in this setting (with extra care taken along the singularities) we obtain:

Theorem 5.2. [BN2] Let $E$ denote a cubic curve and $\mathcal{P}$ the Poincaré sheaf on $E \times E$.

(1) The functor $\mathbb{F}: M \mapsto R p_{2 *}\left(p_{1}^{*} M \otimes \mathcal{P}\right)$ defines an autoequivalence of the bounded derived category of coherent sheaves on $E$ (and likewise for quasicoherent sheaves).

(2) $\mathbb{F}$ induces an equivalence $\mathbb{F}: D^{b}\left(\mathcal{D}_{E}^{\log }\right) \rightarrow D^{b}\left(E^{\natural}\right)$ of bounded derived category of coherent $\mathcal{D}^{\log }$-modules on $E$ and of coherent sheaves on $E^{\natural}$.

\footnotetext{
${ }^{14}$ We consider coherent sheaves on $E^{\natural}$ as $\pi_{*} \mathcal{O}_{E \natural}$-modules on $E$.
} 
The Fourier-Mukai transform is compatible with filtrations on $\mathcal{D}$-modules and $\mathcal{O}_{E^{\natural}}$-modules; as a result, it may be used (as we will see below) to relate framed $\mathcal{D}$-bundles to coherent sheaves on $\bar{E}^{\natural}$.

Remark 5.3. It is useful to note that a degenerate case of the extended Fourier transform gives an autoequivalence of the derived category of the surface $E \times \mathbf{A}^{1}$, i.e. of the derived category of modules over the sheaf of algebras $\mathcal{O}_{E}[s]$. This latter algebra arises as the common degeneration of $\mathcal{D}$ and of the coordinate ring $\mathcal{O}_{E^{\natural}}$.

5.2. Torsion-Free Sheaves and Spectral Sheaves. In this section we would like to describe the effect of the extended Fourier-Mukai transform on framed torsion-free sheaves on $\overline{T_{\hbar}^{*} E}$, that is, on framed $\mathcal{D}$-bundles (see Definition 4.4).

Remark 5.4. The same techniques apply to the commutative limit $E \times \mathbf{P}^{1}$, and can be used to give a new proof of the ADHM classification of framed torsion-free sheaves on $\mathbf{P}^{2}$ by quiver data. Namely, we replace the completion $\mathbf{P}^{2}$ of $\mathbf{A}^{2}$ by the completion $\overline{\mathbb{G}}_{a} \times \mathbf{P}^{1}$, and the Koszul duality and Beilinson spectral sequence by the Fourier-Mukai transform on $\overline{\mathbb{G}}_{a}$.

We will consider torsion-free sheaves on $\overline{T_{\hbar}^{*} E}$, i.e. $\mathcal{D}$-bundles, trivialized along the section $E_{\infty}$ (and more generally in [BN2] sheaves framed by an arbitrary semistable vector bundle $V$ on $E_{\infty}$ of degree 0$)$. We let $\mathfrak{M}_{c_{2}}\left(\overline{T_{\hbar}^{*} E}\right)$ denote the moduli space of these framed $\mathcal{D}$-bundles.

On the Fourier dual side, we will consider coherent sheaves on $\bar{E}^{\natural}$ whose restriction to the section $E_{\infty}$ is identified with the skyscraper sheaf $\mathcal{O}_{b}$ at the basepoint. It follows that such a sheaf has support of dimension at most one. Imposing appropriate normalization conditions, these are precisely the $\mathrm{CM}$ spectral sheaves on $\bar{E}^{\natural}$ from Definition 2.1. These are automatically rank one torsion-free sheaves supported on a curve $\Sigma \subset \bar{E}^{\natural}$ of some degree $n$ over $E$.

The Fourier transform of a framed $\mathcal{D}$-bundle is a priori a complex of sheaves, whose restriction to $E_{\infty}$ is identified with the torsion sheaf $\mathcal{O}_{b}$, the Fourier transform of $\mathcal{O}$. We then prove that the Fourier transform is itself in fact a sheaf, in cohomological degree one, and of pure dimension one, and thus defines a framed spectral sheaf. We obtain the following theorem: 
Theorem 5.5. [BN2] Fix a cubic curve E. The Fourier-Mukai transform induces an isomorphism of the moduli space $\mathfrak{M}_{n}\left(\overline{T_{\hbar}^{*} E}\right)$ of framed $\mathcal{D}$-bundles with $c_{2}=n$ and the Calogero-Moser space $\mathfrak{C M}_{n}(E)$, identified as the space of framed spectral curves of degree $n$ over $E$.

This is the special case of a result describing moduli of $\mathcal{D}$-bundles with general framing at infinity in terms of spectral sheaves on $\bar{E}^{\natural}$. Again, one has an analogous result if one replaces both $\overline{T_{\hbar}^{*} E}$ and $\bar{E}^{\natural}$ by $E \times \mathbf{P}^{1}$ - however, in that case one should naturally replace the commutative analog of $\mathcal{D}$-bundles, i.e. torsion-free sheaves, by the larger class of perverse bundles [BN4].

Thus we obtain a description of the completed phase space of the CalogeroMoser $n$-particle system as a "configuration space of $n$ points on the quantized cotangent bundle". Note that this is a stronger statement than the natural identification of the (uncompleted) phase space of distinct particles with a configuration space on the cotangent bundle, or more generally the birational identification of Hitchin systems with Hilbert schemes of points on (commutative) cotangent bundles, à la Hurtubise $[\mathrm{Hu}]$.

Recall that the Hilbert scheme of $n$ points on $\mathbf{A}^{2}$ has an elementary description, as the set of conjugacy classes of pairs of commuting $n \times n$ matrices $[X, Y]=0$ with a common cyclic vector $\mathbf{C}[X, Y] \cdot v=\mathbf{C}^{n}$ (the associated ideal is the kernel of the projection $\mathbf{C}[X, Y] \rightarrow \mathbf{C}^{n}$ ). Theorem 5.5 in the rational (cuspidal) case $E=\overline{\mathbb{G}}_{a}$, combined with the elementary description of the rational CalogeroMoser phase space, has the following immediate consequence:

Corollary 5.6. The set of isomorphism classes of finitely generated, rank 1, torsion-free right modules for the first Weyl algebra $\mathcal{D}_{\mathbf{A}^{1}}$ is in natural bijective correspondence with the union, over all $n \geq 0$, of the spaces

$$
\mathfrak{C M}_{n}=\left\{(X, Y) \in \mathfrak{g l}_{n} \times \mathfrak{g l}_{n} \mid[X, Y] \in \mathbb{O}\right\} / G L_{n}
$$

This result is immediately implied by combining the two descriptions of the adèlic Grassmannian in [W3] and [CH1], and was proven in [BW2, BGK1, BGK2] using calculations in noncommutative algebraic geometry. Our approach also gives concrete descriptions of ideals in $\mathcal{D}$ over $\mathbb{G}_{m}$ and over an elliptic curve, as well as higher rank versions. 
See also [BN4] for an approach via Koszul duality (in the form of a "fiberwise Beilinson equivalence") that works for curves of arbitrary genus.

Remark 5.7. What we describe in this section is the Fourier transform for $\mathcal{D}$ modules on the projective curve $\overline{\mathbb{G}}_{a}$. There is another Fourier transform for $\mathcal{D}$-modules on $\mathbf{A}^{1}=\mathbb{G}_{a}$, which we discuss in Section 6.2 , and which in particular gives a convenient realization of Wilson's bispectral involution.

5.3. The KP/CM Correspondence. As we have mentioned earlier, an important special class of solutions to the KP equation, first investigated by Krichever [Kr1, Kr2] and the Chudnovskys [CC], consists of functions $u=u(t, x, y)$ which are rational, trigonometric or elliptic functions of the first $\mathrm{KP}$ time $t$, i.e. extend to rational functions on the additive group $\mathbf{C}$, the multiplicative group $\mathbf{C}^{\times}$ or an elliptic curve $E$. An analogous question for the KP hierarchy (studied in [Shi, W3] in the rational case) seeks to describe KP Lax operators $L$ which are rational, trigonometric or elliptic as functions of the first KP time, namely those $L$ whose orbit under the vector field $\frac{\partial}{\partial t}$ closes up to (a Zariski open subset of) an additive, multiplicative or elliptic group.

A complete description of the rational, trigonometric and elliptic solitons of KP follows from the Fourier-Mukai transform, specifically Theorem 5.5 identifying framed $\mathcal{D}$-bundles with second Chern class $n$ with the $n$th Calogero-Moser phase space. Indeed, we have identified KP Lax operators with micro-opers, which are framed $\mathcal{D}$-bundles with an additional endomorphism $\partial_{M}$ of their microlocalizations. As we explain in [BN2, Section 3.6.1], the collection of such additional choices of microlocal endomorphisms assembles into a Lie algebroid, the KP algebroid, over the moduli space of $\mathcal{D}$-bundles. Similarly, the Hamiltonian flows on the CM phase space are invariantly captured by the action of a Lie algebroid, the Lie algebroid of tweaking flows [BN2, BN3] on the CM phase space.

Theorem 5.5 then allows us to identify these two Lie algebroids, intertwining their actions. Thus, we identify the flows on micro-opers, i.e. meromorphic KP Lax operators, with the flows in the Calogero-Moser particle system:

Theorem 5.8 ([BN2], Theorem 3.25). Let E denote an arbitrary cubic curve.

(1) The isomorphism between the moduli space of $\mathcal{D}$-bundles on $E$ with $c_{2}=$ $n$ and the nth Calogero-Moser space $\mathfrak{C M}_{n}(E)$ provided by Theorem 5.5 
identifies the algebroid of KP flows with the algebroid of Calogero-Moser flows.

(2) This isomorphism identifies the positions of the cusps of a $\mathcal{D}$-bundle (or poles of a KP Lax operator) and of the corresponding Calogero-Moser particles.

The proof of the first part of the theorem is immediate from the descriptions of both KP flows and CM flows, via the actions of the KP and CM algebroids, as modifications of sheaves along the divisor at infinity. Indeed, let $\mathcal{L}$ denote a CM spectral sheaf, supported on a curve $\Sigma \subset \bar{E}^{\natural}$ with unique, transversal intersection $o$ with the curve $E_{\infty}$, and $M$ the $\mathcal{D}$-bundle corresponding to $\mathcal{L}$. The $\mathrm{CM}$ flows act on $\mathcal{L}$ through modifications at the point $o$ ("tweaking flows"): restricting $\mathcal{L}$ to the formal punctured neighborhood of $E_{\infty}$, we obtain a sheaf whose endomorphism algebra is isomorphic to the field of Laurent series; the polar parts of Laurent series act as infinitesimal deformations of $\mathcal{L}$. This commutative algebra of endomorphisms is identified by the Fourier-Mukai transform with the algebra of endomorphisms of the $\mathcal{E}$-module $M_{\mathcal{E}}=M \otimes_{\mathcal{D}} \mathcal{E}$ (the restriction of $M$ to the "formal punctured neighborhood" of the section $E_{\infty} \subset \overline{T_{\hbar}^{*} E}$ ). But we have described the KP flows on micro-opers precisely through the action of these endomorphisms, more specifically of endomorphisms induced by powers of the endomorphism $\partial_{M}$, so that the identification of the flows is straightforward.

The statement about locations of the poles is also easy using the FourierMukai transform: recall (equation 2.3) that a configuration of $n$ Calogero-Moser particles with distinct positions is described, in the Hitchin system description, by a spectral sheaf that pushes forward to the direct sum of line bundles $\mathcal{O}\left(q_{i}-o\right)$ on $E$. These line bundles correspond to the $n$ points $q_{i}$ under the Fourier transform, and these points determine the positions at which one creates cusps in the microoper.

Remark 5.9. It is instructive to compare the above description of elliptic solitons with the $\mathcal{D}$-module description of the Krichever construction due to Nakayashiki and Rothstein [N1, N2, Ro1, Ro2]. Assume for simplicity that the micro-oper $M$ on $E$ corresponds to a spectral sheaf which is a line bundle $\mathcal{L}$ on a smooth spectral curve $\Sigma \rightarrow E$. The dual to the map Jac $\Sigma \rightarrow E$ is a map $E \rightarrow \operatorname{Jac} \Sigma$, which is tangent to $\Sigma \hookrightarrow$ Jac $\Sigma$ at the point $x=\Sigma \cap \bar{o}$-in other words $\Sigma \rightarrow E$ is a tangential cover $([\mathrm{TV} 1, \mathrm{DM}])$. The $\mathcal{D}$-bundle $M$ on $E$ is then the restriction 
to $E$ of the Krichever $\mathcal{D}$-module $\operatorname{Krich}(\mathcal{L})$ on $\operatorname{Jac} \Sigma$, obtained (as explained in [Ro2]) as the Fourier-Mukai transform of the Abel-Jacobi pushforward of $\mathcal{L}(* x)$ from $\Sigma$ to Jac $\Sigma$. The cusps of the $\mathcal{D}$-bundle $M$ correspond to the intersection points of $E$ with the theta divisor of Jac $\Sigma$.

\section{Further TOPICS}

In this section we sketch some further applications of the techniques described in the previous directions.

\subsection{The Multicomponent KP/Spin Calogero-Moser Correspondence.} The description and extension of the $\mathrm{KP} / \mathrm{CM}$ correspondence outlined in this paper are worked out in greater generality in the paper [BN2]. In particular, we extend the correspondence to a relation between the multicomponent KP hierarchy and the spin generalizations of the Calogero-Moser system, generalizing and refining the results of [BBKT, T1] in several directions.

The multicomponent KP hierarchy is the matrix generalization of the KP hierarchy, where we replace the algebra $\mathcal{E}$ with $\mathfrak{g l}_{n}(\mathcal{E})$, the algebra of $n$ by $n$ matrices over $\mathcal{E}$, and the Sato Grassmannian with the $n$-component $\operatorname{Grassmanian} \operatorname{Gr}\left(\mathcal{V}^{\oplus n}\right)$ - see, for example, [LM, P] for more details. The geometric interpretation of KP in terms of micro-opers is extended to this setting by replacing $\mathcal{D}$-line bundles by higher rank $\mathcal{D}$-bundles, with framing by a general semistable vector bundle of degree 0. Higher rank micro-opers are a Higgs refinement of this structure, namely they carry actions of commutative algebras of matrix microdifferential operators. On the Fourier dual side one obtains general spectral curves in $\bar{E}^{\natural}$, whose geometry is determined by the framing conditions at infinity. In the case of trivially framed $\mathcal{D}$-bundles, we obtain precisely the spin generalization of the CalogeroMoser system, $[\mathrm{GH}]$, and thus obtain an extension of Wilson's description of pole collisions to a spin $\mathrm{CM}$ /multicomponent $\mathrm{KP}$ correspondence, in rational, trigonometric and elliptic settings. For other kinds of framings we obtain "multicolored" spin Calogero-Moser systems, describing pole motion of different reductions of multicomponent KP.

Among the reductions of multicomponent KP are the 2D Toda lattice hierarchies. Using a difference analog of the techniques of this paper, we develop in 
[BN5] an extension of the geometric picture described above to a Toda/RuijsenaarsSchneider correspondence, extending the results of [KrZ].

6.2. Bispectrality. Let us recall the notion of bispectrality of differential operators, introduced by Duistermaat and Grünbaum [DG]. Differential operators $L\left(t, \partial_{t}\right)$ and $\Lambda\left(z, \partial_{z}\right)$ in two variables $t, z$ are said to be bispectral if there exists a function $\psi(z, t)$ which is simultaneously a parametric family of eigenfunctions for $L$ and $\Lambda$,

$$
L \cdot \psi(z, t)=f(z) \psi(z, t), \quad \Lambda \cdot \psi(z, t)=g(t) \psi(z, t)
$$

with nonconstant $f, g$. G. Wilson [W1] discovered a remarkable symmetry of the collection of rational solitons (decaying at infinity) of KP, the bispectral involution, which expresses the bispectrality of these solutions. Namely to each point $W$ in Wilson's adèlic Grassmannian (the parameter space of the rational solitons) is associated a Baker function $\psi_{W}(z, t)$, depending on the spectral parameter $z$ and the KP times $t$, which is a parametric family of joint eigenfunctions for a commutative ring of differential operators (this is the ring of functions on the associated spectral curve). The bispectral involution $W \mapsto b(W)$ is characterized by the property that it interchanges the spectral parameter with the first KP time, $\psi_{b(W)}(z, t)=\psi_{W}(t, z)$ (though it is by no means clear from this characterization that the desired involution exists). It follows that the KP solution corresponding to $W$ is bispectral: it is an eigenfunction for ordinary differential operators both $z$ and in $t$. In [BW1], Berest and Wilson use the Cannings-Holland bijection between the adèlic Grassmannian and ideals in the Weyl algebra $\mathcal{D}\left(\mathbf{A}^{1}\right)$ to give a simpler description of the bispectral involution and derivation of its main properties. Namely they identify it with the action of the antiautomorphism

$$
\mathbb{F}: \mathcal{D}\left(\mathbf{A}^{1}\right) \rightarrow \mathcal{D}\left(\mathbf{A}^{1}\right), \quad t \mapsto \partial_{t}, \partial_{t} \mapsto t
$$

of the Weyl algebra, i.e. the geometric Fourier transform composed with the map $t \mapsto-t .{ }^{15}$

Our description of the rational KP solutions in terms of micro-opers gives a simple conceptual framework for bispectrality in rational KP. The crucial observation is that micro-opers on $\mathbf{A}^{1}$ define $\mathrm{KP}$ solutions in two independent fashions, as was explained in Section 4.5, which are interchanged by the geometric

\footnotetext{
${ }^{15}$ See the beautiful survey articles [W2, W4, W5] for overviews of the work of Wilson and Berest-Wilson.
} 
Fourier transform. More precisely, we consider micro-opers on $\mathbf{P}^{1}$ that are locally free as $\mathcal{D}$-modules near $\infty$-such micro-opers are in bijection with points of $\mathfrak{G r}^{a d}$. We then define natural embeddings of this space in the Sato Grassmannians $\operatorname{Gr}\left(\mathbf{C}\left(\left(\partial_{t}^{-1}\right)\right)\right)$ and $\operatorname{Gr}\left(\mathbf{C}\left(\left(t^{-1}\right)\right)\right)$. The first map from micro-opers to the Sato Grassmannian uses Theorem 4.7: a micro-oper on $\mathbf{P}^{1}$, when expanded near $z=0$, gives a KP Lax operator and thus by Sato's construction a point of the Grassmannian $\operatorname{Gr}\left(\mathbf{C}\left(\left(\partial_{t}^{-1}\right)\right)\right)$. The second map uses a micro-oper to define a torsion-free sheaf on a cuspidal quotient of $\mathbf{A}^{1}$, which (using natural trivialization data at $\infty)$ defines a subspace of $\mathbf{C}\left(\left(t^{-1}\right)\right)$, i.e. a point in $\operatorname{Gr}\left(\mathbf{C}\left(\left(t^{-1}\right)\right)\right)$. The geometric Fourier transform, which defines an autoequivalence of the category of $\mathcal{D}$-modules on $\mathbf{A}^{1}$, induces an involution of this space of micro-opers on $\mathbf{P}^{1}$. The Fourier transform also identifies $\mathbf{C}\left(\left(z^{-1}\right)\right)$ and $\mathbf{C}\left(\left(\partial_{t}^{-1}\right)\right)$, their two Grassmannians, and the collections of KP flows on these Grassmannians given by the action of $\mathbf{C}\left[\partial_{t}\right]$ and $\mathbf{C}[t]$, respectively. It is easy to see that this identification interchanges the role of the spectral parameter and of the first KP time, and hence gives the bispectrality of the rational KP solutions.

6.3. $\mathcal{W}_{1+\infty}$ and the Adelic Grassmannian. In [BN6] we describe vertex algebra structures associated to $\mathcal{D}$-bundles through the adelic Grassmannian. Namely, for a finite set $I$, the functor of flat families of $\mathcal{D}$-bundles trivialized away from $I$ points forms an ind-scheme of ind-finite type over $X^{I}$. The directed system of these spaces over $I$, together with factorization isomorphisms describing the decomposition of these spaces with respect to disjoint unions $I \amalg J$, make the adelic Grassmannian into a factorization ind-scheme ([BD2]). Moreover the (twisted) delta-functions at the trivial bundle form a factorization algebra, which we identify with the $\mathcal{W}_{1+\infty}$-vertex algebra (the enveloping vertex algebra of the central extension of the Lie algebra $\mathcal{D}(\mathcal{K})$ ). It follows that the moduli stacks of $\mathcal{D}$-bundles are uniformized by $\mathcal{W}_{1+\infty}$-vertex algebra, and that we obtain a localization for representations of the latter algebra (in other words a definition of sheaves of twisted conformal blocks). If we replace trivializations of $\mathcal{D}$-bundles by identifications with some nontrivial $\mathcal{D}$-bundle, we obtain a new continuous family of chiral algebras, parametrized by geometry of cusps of $\mathcal{D}$-bundles, all of which localize on $\mathcal{D}$-moduli spaces. This provides a starting point for the development of the so-called " $\mathcal{W}$-geometry" from string theory. 
6.4. Hilbert Schemes and Separation of Variables. The space $\mathfrak{C M}_{n}(E)$ of Calogero-Moser spectral sheaves may be identified birationally with the Hilbert scheme of $n$ points on $\bar{E}^{\natural}$, as in [Hu] and in [GNR], where this description is interpreted as Sklyanin's separation of variables. A remarkable feature of the KP/CM correspondence, however, is that on the $\mathcal{D}$-side the entire space $\mathfrak{C M}_{n}(E)$ is realized biregularly as a Hilbert scheme of points (moduli of rank one torsion-free sheaves) on the noncommutative cotangent bundle of $E$. The description of positions and "momenta" of the cusps of a generic $\mathcal{D}$-bundle $M$ (the latter being coordinates on the one-dimensional Schubert cells in the adelic Grassmannian), which give the Calogero-Moser particles, determine a canonical birational identification with a Hilbert scheme of points on a (commutative) twisted cotangent bundle. This picture demonstrates that in order to allow collisions, the proper completion of the phase space is the noncommutative Hilbert scheme, to which the flows extend, rather than the commutative one. Also our description of the noncommutative separation of variables as a Fourier-Mukai transform establishes the speculation of [GNR] that separation of variables is a T-duality (see also [KS]).

6.5. Noncommutative Instantons. $\mathcal{D}$-bundles on a curve $X$, namely holomorphic bundles on the quantized cotangent bundle $T_{\hbar}^{*} X$ (see Section 4.1), are part of the subject of noncommutative gauge theory $([\mathrm{Ne} 2])$ : one expects a noncommutative version of the Donaldson-Uhlenbeck-Yau Theorem to identify $\mathcal{D}$-bundles with noncommutative Yang-Mills instantons. In the rational case, i.e. on the quantum plane $T_{\hbar}^{*} \mathbf{A}^{1}$, such a correspondence is provided a posteriori by the explicit description of $\mathcal{D}$-bundles by matrices in Corollary 5.6, and its higher rank generalizations. Namely, $\mathcal{D}$-bundles on $\mathbf{A}^{1}$ are classified by the deformed ADHM data, which was shown by Nekrasov and Schwarz [NeS] to describe the YangMills instantons on noncommutative $\mathbf{R}^{4}$ (see also [W3, KKO, BrNe, BGK2]). These noncommutative instantons were proposed as a gauge-theoretic substitute for the moduli spaces of torsion-free sheaves, resolving the pointlike instanton singularities of the commutative instanton moduli spaces on $\mathbf{P}^{2}$. The moduli of noncommutative instantons (i.e. $\mathcal{D}$-bundles on $\mathbf{A}^{1}$ ) are algebraically nontrivial (albeit diffeomorphic) deformations of the classical instanton moduli spaces. More generally, bundles on $T_{\hbar}^{*} X$ (i.e. $\mathcal{D}$-bundles on $X$ ) appear to be a good algebraic model for the systems of D0 branes bound to a D4 brane by a background $B$-field [NeS, KS, GNR]. While the $\mathcal{D}$-bundles are projective, making possible their noncommutative instanton interpretation (e.g. $[\mathrm{Fu}]$ ), they are not locally 
free, with singularities (cusps) at special points which correspond to the position and momenta of the corresponding Calogero-Moser particles, so that the D0 branes in the D4 brane are naturally modeled by a many-body system. The noncommutativity of $T_{\hbar}^{*} X$, however, masks these singularities within projective modules.

\section{REFERENCES}

[AVV] M.J. Asensio, M. Van den Bergh, and F. van Oystaeyen, A new algebraic approach to microlocalization of filtered rings, Trans. Amer. Math. Soc. 316 (1989), no. 2, 537-553.

[AMM] H. Airault, H. P. McKean, and J. Moser, Rational and elliptic solutions of the Kortewegde Vries equation and a related many-body problem, Comm. Pure Appl. Math. 30 (1977), no. 1, 95-148.

[AKV] A. Akhmetshin, I. Krichever and Y. Volvovski, Elliptic families of solutions of the Kadomtsev-Petviashvili equations and the field elliptic Calogero-Moser system, Funk. Anal. i Prilozhen. 36 (2002), no. 4, 1-17. arXiv:hep-th/0203192.

[AMP] A. Álvarez, J. Muñoz and F. Plaza, The algebraic formalism of soliton equations over arbitrary base fields. In Workshop on Abelian Varieties and Theta Functions (Morelia 1996), Aportaciones Mat. Investig. 13, 3-40, Soc. Mat. Mexicana, 1998. alggeom/9606009.

[BB] O. Babelon and D. Bernard, The Sine-Gordon solitons as a $N$-body problem, Phys. Lett. B 317 (1993), no. 3, 363-368. arXiv:hep-th/9309154.

[BBKT] O. Babelon, E. Billey, I. Krichever and M. Talon, Spin generalization of the CalogeroMoser system and the matrix KP equation. In Topics in Topology and Mathematical Physics, Amer. Math. Soc. Trans. Ser.2 170, 83-119, Amer. Math. Soc., Providence, RI, 1995.

[BGK1] V. Baranovsky, V. Ginzburg, and A. Kuznetsov, Quiver varieties and a noncommutative $\mathbf{P}^{2}$, Compositio Math. 134 (2002), no. 3, 283-318. arXiv:math.AG/0103068.

[BGK2] V. Baranovsky, V. Ginzburg, and A. Kuznetsov, Wilson's Grassmannian and a noncommutative quadric, Int. Math. Res. Not. 21 (2003), 1155-1197. arXiv:math.AG/0203116.

[BL] A. Beauville and Y. Laszlo, Conformal blocks and generalized theta functions, Comm. Math. Phys. 164 (1993), 385-419. arXiv:math.AG/9309003.

[BD1] A. Beilinson and V. Drinfeld, Quantization of Hitchin's integrable system and Hecke eigensheaves, in preparation, available at www.math.utexas.edu/ benzvi.

[BD2] A. Beilinson and V. Drinfeld, Chiral algebras, in preparation, available at www.math.utexas.edu/ benzvi.

[Be] D. Bennequin, Hommage à Jean-Louis Verdier: Au jardin des systèmes intégrables. In Integrable Systems: The Verdier Memorial Conference, Prog. Math. 115 (1993), 1-36.

[BF] D. Ben-Zvi and E. Frenkel, Spectral curves, opers and integrable systems, Inst. Hautes Études Sci. Publ. Math. 94 (2001), 87-159. arXiv:math.AG/9902068. 
[BN1] D. Ben-Zvi and T. Nevins, Cusps and D-modules, J. Amer. Math. Soc. 17 (2004), no. 1, 155-179. arXiv:math.AG/0212094.

[BN2] D. Ben-Zvi and T. Nevins, D-bundles and integrable hierarchies, arXiv:math/0603720.

[BN3] D. Ben-Zvi and T. Nevins, Flows of Calogero-Moser systems, Int. Math. Res. Not., to appear, arXiv:math/0603722.

[BN4] D. Ben-Zvi and T. Nevins, Perverse bundles and Calogero-Moser spaces, arXiv:math/0610097.

[BN5] D. Ben-Zvi and T. Nevins, Toda lattice hierarchy and noncommutative geometry, in preparation.

[BN6] D. Ben-Zvi and T. Nevins, $\mathcal{W}_{\infty}$-algebras and $\mathcal{D}$-moduli spaces, in preparation.

[BW1] Y. Berest and G. Wilson, Automorphisms and ideals of the Weyl algebra, Math. Ann. 318 (2000), 127-147. arXiv:math.QA/0102190.

[BW2] Y. Berest and G. Wilson, Ideal classes of the Weyl algebra and noncommutative projective geometry, With an appendix by Michel Van den Bergh, Int. Math. Res. Not. 2 (2002), 1347-1396. arXiv:math.AG/0104248.

[BrH] H. Braden and A. Hone, Affine Toda solitons and systems of Calogero-Moser type, Phys. Lett. B380 (1996), 296-302. arXiv:hep-th/9603178.

[BrK] H. Braden and I. Krichever, eds., Integrability: The Seiberg-Witten and Whitham Equations. Gordon and Breach, 2000, 276pp.

[BrNe] H. Braden and N. Nekrasov, Instantons, Hilbert schemes and integrability. In Integrable structures of exactly solvable two-dimensional models of quantum field theory (Kiev, 2000), NATO Sci. Ser. II Math. Phys. Chem. 35, 35-54, Kluwer Acad. Publ., Dordrecht, 2001. arXiv:hep-th/0103204.

[CH1] R. Cannings and M. Holland, Right ideals of rings of differential operators, J. Algebra 167 (1994), 116-141.

[CH2] R. Cannings and M. Holland, Limits of compactified Jacobians and $\mathcal{D}$-modules on smooth projective curves, Adv. Math. 135 (1998), 287-302.

[CC] D.V. Chudnovsky and G.V. Chudnovsky, Pole expansions of nonlinear partial differential equations, Il Nuovo Cimento 40B (1977), 339-353.

[DM] R. Donagi and E. Markman, Spectral covers, algebraically completely integrable Hamiltonian systems, and moduli of bundles. In Integrable systems and quantum groups, Lecture Notes in Math. 1620, Springer-Verlag, New York, 1995. alg-geom/9507017.

[DW] R. Donagi and E. Witten, Supersymmetric Yang-Mills theory and integrable systems, Nucl. Phys. B460 (1996), 299-334. arXiv:hep-th/9510101.

[Dr] V. Drinfeld, DG quotients of DG categories, J. Algebra 272 (2004), no. 2, 643-691, arXiv:math.KT/0210114.

[DS] V. Drinfeld and V. Sokolov, Lie algebras and equations of Korteweg-deVries type, J. Soviet Math. 30 (1985), 1975-2035.

[DG] J.J. Duistermaat and F.A. Grünbaum, Differential equations in the spectral parameter, Comm. Math. Phys. 93 (1984), 453-460.

[EO] B. Enriquez and A. Odesskii, Quantization of canonical cones of algebraic curves, Ann. Inst. Fourier (Grenoble) 52 (2002), no. 6, 1629-1663. arXiv:math.QA/0112148. 
[FM] R. Friedman and J. Morgan, Holomorphic principal bundles over elliptic curves III: singular curves and fibrations, preprint. arXiv:math.AG/0108104.

[Fu] K. Furuuchi, Instantons on noncommutative $\mathbf{R}^{4}$ and projection operators, Progr. Theoret. Phys. 103 (2000), no. 5, 1043-1068. arXiv:hep-th/9912047.

[GW] F. Gesztesy and R. Weikard, Elliptic algebro-geometric solutions of the KdV and AKNS hierarchies - an analytic approach, Bull. Amer. Math. Soc. (N.S.) 35 (1998), no. 4, 271-317.

[GH] J. Gibbons and T. Hermsen, A generalization of the Calogero-Moser system, Physica 11D (1984), 337-348.

[GN] A. Gorsky and N. Nekrasov, Elliptic Calogero-Moser system from two-dimensional current algebra, preprint. hep-th/9401021.

[GNR] A. Gorsky, N. Nekrasov and V. Rubtsov, Hilbert schemes, separated variables and D-branes, Comm. Math. Phys. 222 (2001), no. 2, 299-318. arXiv:hep-th/9901089.

[Hi] N. Hitchin, Stable bundles and integrable systems, Duke Math. J. 54 (1990), 91-114.

[Hu] J. Hurtubise, Integrable systems and algebraic surfaces, Duke Math J. 83 (1996), 19-50.

[KKO] A. Kapustin, A. Kuznetsov and D. Orlov, Noncommutative instantons and twistor transform, Comm. Math. Phys. 221 (2001), no. 2, 385-432. arXiv:hep-th/0002193.

[KS] A. Kapustin and S. Sethi, The Higgs branch of impurity theories, Adv. Theor. Math. Phys. 2 (1998), no. 3, 571-591. arXiv:hep-th/9804027.

[Ka] M. Kashiwara, D-modules and microlocal calculus, translated from the 2000 Japanese original by Mutsumi Saito. Translations of Mathematical Monographs 217, Iwanami Series in Modern Mathematics. American Mathematical Society, Providence, RI, 2003. $\mathrm{xvi}+254 \mathrm{pp}$.

[KKS] D. Kazhdan, B. Kostant and S. Sternberg, Hamiltonian group actions and dynamical systems of Calogero type, Comm. Pure Appl. Math 31 (1978), 481-507.

[Kr1] I. M. Krichever, On rational solutions of Kadomtsev-Petviashvilii equation and integrable systems of $N$ particles on line, Funct. Anal i Pril. 12 (1978), no. 1, 76-78.

[Kr2] I. M. Krichever, Elliptic solutions of the Kadomtsev-Petviashvilii equations and integrable systems of particles, Funct. Anal. Appl. 14 (1980), no. 4, 282-290.

[Kr3] I. M. Krichever, Elliptic solutions to difference non-linear equations and nested Bethe ansatz equations. In Calogero-Moser-Sutherland models (Montréal, QC, 1997), 249271, CRM Ser. Math. Phys., Springer, New York, 2000. arXiv:solv-int/9804016.

[Kr4] I. M. Krichever, Elliptic analog of the Toda lattice, Int. Math. Res. Not. 8 (2000), 383-412. arXiv:hep-th/9909224.

[Kr5] I. M. Krichever, Vector bundles and Lax equations on algebraic curves, Comm. Math. Phys. 229 (2002), 229-269. arXiv:hep-th/0108110.

[KrZ] I. M. Krichever and A. Zabrodin, Spin generalization of the Ruijsenaars-Schneider model, nonabelian 2D Toda chain, and representations of Sklyanin algebra, (Russian) Uspekhi Mat. Nauk 50 (1995), no. 6(306), 3-56; translation in Russian Math. Surveys 50 (1995), no. 6, 1101-1150. arXiv:hep-th/9505039.

[La1] G. Laumon, Sur la categorie derivée des $\mathcal{D}$-modules filtrées. In Algebraic geometry (Tokyo/Kyoto, 1982), Lecture Notes in Math. 1016, 151-237, Springer, Berlin, 1983. 
[La2] G. Laumon, Transformation de Fourier généralisée, preprint. alg-geom/9603004.

[LB] L. Le Bruyn, Moduli spaces for right ideals of the Weyl algebra. J. Algebra 172 (1995), no. $1,32-48$.

[LM] Y. Li and M. Mulase, Prym varieties and integrable systems, Comm. Anal. and Geom. 5 (1997), 279-332. arXiv:math.AG/9203002.

[Ma] Y. Manin, Sixth Painlevé equation, universal elliptic curve, and mirror of $\mathbf{P}^{2}$. In Geometry of differential equations, Amer. Math. Soc. Transl. Ser. 2 186, 131-151, Amer. Math. Soc., Providence, RI, 1998. arXiv:math.AG/9605010.

[Muk] S. Mukai, Duality between $D(X)$ and $D(\hat{X})$ with its application to Picard sheaves, Nagoya Math. J. 81 (1981), 153-175.

[Mu1] M. Mulase, Complete integrability of the Kadomtsev-Petviashvili equation, Adv. in Math. 54 (1984), 57-66.

[Mu2] M. Mulase, Algebraic theory of the KP equations. In Perspectives in mathematical physics, Conf. Proc. Lect. Notes Math. Phys. III (1994), 151-217.

[N1] A. Nakayashiki, Structure of Baker-Akhiezer modules of pricipally polarized abelian varieties, commuting partial differential operators and associated integrable systems, Duke Math J. 62 (1991), 315-358.

[N2] A. Nakayashiki, Commuting partial differential operators and vector bundles over abelian varieties, Amer. J. Math. 116 (1994), 65-100.

[Ne1] N. Nekrasov, Holomorphic bundles and many-body systems, Comm. Math. Phys. 180 (1996), no. 3, 587-603. arXiv:hep-th/9503157.

[Ne2] N. Nekrasov, Trieste lectures on solitons in noncommutative gauge theories. In Superstrings and related matters (Trieste, 2000), 141-205, World Sci. Publishing, River Edge, NJ, 2001. arXiv:hep-th/0011095.

[NeS] N. Nekrasov and A. Schwarz, Instantons on noncommutative $\mathbf{R}^{4}$ and $(2,0)$ superconformal six-dimensional theory, Comm. Math. Phys. 198 (1998), 689-703. arXiv:hepth/9802068.

[P] F. Plaza Martin, Algebraic solutions of the multicomponent KP hierarchy, J. Geom. Phys. 36 (2000), no. 1-2, 1-21. arXiv:math.AG/9907069.

[PRo] A. Polishchuk and M. Rothstein, Fourier transform for $\mathcal{D}$-algebras I, Duke Math. J. 109 (2001), no. 1, 123-146. arXiv:math.AG/9901009.

[Ro1] M. Rothstein, Connections on the total Picard sheaf and the KP hierarchy, Acta Appl. Math. 42 (1996), 297-308.

[Ro2] M. Rothstein, Sheaves with connection on abelian varieties, Duke Math J. 84 (1996) No.3, 565-598. arXiv:math.AG/9602023.

[S] M. Sato, The KP hierarchy and infinite-dimensional Grassmann manifolds. In Theta functions-Bowdoin 1987, Part 1 (Brunswick, ME, 1987), Proc. Sympos. Pure Math. 49, Part 1, 51-66, Amer. Math. Soc., Providence, RI, 1989.

[SW] G. Segal and G. Wilson, Loop groups and equations of KdV type, Inst. Hautes Études Sci. Publ. Math. 61 (1985), 5-65. 
[Sh] P. Schapira, Microdifferential systems in the complex domain. Grundlehren der Mathematischen Wissenschaften [Fundamental Principles of Mathematical Sciences], 269. Springer-Verlag, Berlin, 1985. x+214 pp.

[Shi] T. Shiota, Calogero-Moser hierarchy and KP hierarchy, J. Math. Phys. 35 (1994), 5844-5849. arXiv:hep-th/9402021.

[SS] S. P. Smith and J. T. Stafford, Differential operators on an affine curve, Proc. London Math. Soc. (3) 56 (1988), 229-259.

[Sp] T. Springer, Micro-localization algébrique. In Séminaire d'algèbre Paul Dubreil et Marie-Paule Malliavin, 36ème année (Paris, 1983-1984), Lecture Notes in Math. 1146, 299-316, Springer, Berlin, 1985.

[St] J.T. Stafford, Noncommutative projective geometry. In Proceedings of the International Congress of Mathematicians, Vol. II (Beijing, 2002), 93-103, Higher Ed. Press, Beijing, 2002. arXiv:math.RA/0304210.

[StV] J.T. Stafford and M. Van den Bergh, Noncommutative curves and noncommutative surfaces, Bull. Amer. Math. Soc. (N.S.) 38 (2001), no. 2, 171-216. arXiv:math.RA/9910082.

[T1] A. Treibich, Matrix elliptic solitons, Duke Math. J. 90 (1997), no. 3, 523-546.

[T2] A. Treibich, Difference analogs of elliptic KdV solitons and Schrödinger operators, Int. Math. Res. Not. 6 (2003), 313-360.

[TV1] A. Treibich and J.-L. Verdier, Solitons elliptiques (with an appendix by J. Oesterlé). In The Grothendieck Festschrift Vol. 3, Prog. Math. 88, 437-480, Birkhaüser, Boston, 1990 .

[TV2] A. Treibich and J.-L. Verdier, Variétés de Kritchever des solitons elliptiques de KP. In Proceedings of the Indo-French Conference on Geometry (Bombay, 1989), 187-232, Hindustan Book Agency, Delhi, 1993.

[W1] G. Wilson, Bispectral commutative ordinary differential operators, J. reine angew. Math. 442 (1993), 177-204.

[W2] G. Wilson, Bispectral algebras of ordinary differential operators. In The Bispectral Problem, Montréal 1997. CRM Proc. Lect. Notes. 14, 131-137, Amer. Math. Soc., Providence, RI, 1998.

[W3] G. Wilson, Collisions of Calogero-Moser particles and an adelic Grassmannian, Invent. Math. 133 (1998), 1-41.

[W4] G. Wilson, Bispectral symmetry, the Weyl algebra and differential operators on curves, Proc. Steklov Inst. Math. 225 (1999), 141-147.

[W5] G. Wilson, The complex Calogero-Moser and KP systems. In Calogero-MoserSutherland models (Montréal, QC, 1997), 539-548, CRM Ser. Math. Phys., Springer, New York, 2000. 
David Ben-Zvi

Department of Mathematics

University of Texas

Austin, TX 78712-0257

E-mail: benzvi@math.utexas.edu

Thomas Nevins

Department of Mathematics

University of Illinois at Urbana-Champaign

Urbana, IL 61801

E-mail: nevins@uiuc.edu 